\title{
PERTURBATIONS OF \\ NON SELF-ADJOINT STURM-LIOUVILLE PROBLEMS, WITH APPLICATIONS TO HARMONIC OSCILLATORS*
}

\author{
LAURENCE NEDELEC ${ }^{\dagger}$
}

\begin{abstract}
We study the behavior of the limit of the spectrum of a non self-adjoint SturmLiouville operator with analytic potential as the semi-classical parameter $h \rightarrow 0$. We get a good description of the spectrum and limit spectrum near $\infty$. We also study the action of one special perturbation of the operator (adding a Heaviside function), and prove that the limit spectrum is very unstable. As an illustration we describe the limit spectrum as $h \rightarrow 0$ for $P^{h}=-h^{2} \Delta+i x^{2}$ and the effect of this perturbation.
\end{abstract}

Key words. Eigenvalues, Non self-adjoint operators, Sturm-Liouville theory

AMS subject classifications. 34L05 34D10 34L40

1. Introduction. This paper is devoted to non self-adjoint Sturm-Liouville problems. We study the spectrum of the 1-dimensional, semiclassical Schrödinger operator on $L^{2}([-1,1])$ with Dirichlet boundary condition, given by

$$
H^{h}=-h^{2} \frac{d}{d x^{2}}+V(x) .
$$

The potential $V$ is a complex valued function on $[-1,1]$, which extends holomorphically to some domain in $\mathbb{C}$. The boundary value at \pm 1 play no special rule but are fixed to avoid more notation.

The study of such operators is motivated by the Orr-Sommerfeld equation with linear profile [6] or by the non linear Zakharov-Shabat eigenvalue problem, cf. work of Miller [13].

As an application we will focus on the case where

$$
V(x)=i x^{2},
$$

and shall write $P^{h}$ for the corresponding operator. From this one could also study the slightly more general case $-h^{2} \frac{d}{d x^{2}}+e^{d} x^{2}, d \in \mathbb{C}$ using a change of variable. The spectrum of this operator on $\mathbb{R}$ (without Dirichlet condition) was analyzed by Davies [2], cf. also the recent work of Hitrik [9].

It is well known that the spectrum of a non self-adjoint operator is unstable under perturbation of the operator. This motivates the introduction of the pseudo-spectrum, which has now been studied by many people, particularly Trefethen (who maintains the web archive

http:web.comlab.ox.ac.uk/projects/pseudospectra) and Davies [3],[4]; we note also the recent paper of Denker, Sjöstrand and Zworski [5].

We also consider the following perturbation of $H$, non smooth:

For $\beta \in(-1,1)$ and $\delta \geq 0$, let $H_{\delta, \beta}$

$$
H_{\delta, \beta}^{h}=-h^{2} \frac{d}{d x^{2}}+V_{\delta, \beta}(x), \quad V_{\delta, \beta}(x)=\left\{\begin{array}{ll}
V(x)+i \delta, & x>\beta \\
V(x)-i \delta, & x<\beta
\end{array},\right.
$$

\footnotetext{
*Received December 13, 2004; accepted for publication August 24, 2006.

$\dagger$ †.A.G.A., Institut Galilée, Université de Paris Nord, av. J.B. Clement, F-93430 Villetaneuse, France; IUFM de l'academie de Rouen, France (nedelec@math.univ-paris13.fr). Supported in part by the FIM of ETHZ.
} 
with domain

$$
\mathcal{D}_{\delta, \beta}=\left\{u \in L^{2}([-1,1]), u^{\prime \prime} \in L^{2}([-1,1]), u(-1)=u(1)=0,\right\} .
$$

Notice that $H_{0, \beta}^{h}=H^{h}$.

Redparth [14] has obtained similar results as here for one special class of potential i.e. arbitrary piecewise linear complex-valued potentials, a class which includes perturbations as above of the operator $-h^{2} \Delta+i x$ using the Airy function.

We focus on the semi classical limit, and denote by $\lim _{h} \operatorname{Sp}\left(H_{\delta}^{h}\right)$ the set of all values of $E$ which arise as limits as $h \rightarrow 0$ of some sequence of eigenvalues of $H_{\delta}^{h}$. Our goal is to :

- Described the set $\lim _{h} \operatorname{Sp}\left(H_{\delta}^{h}\right)$ for $\delta=0$ and $\delta \neq 0$.

- Find a "Bohr-Sommerfield" condition that describe $\operatorname{Sp}\left(H_{\delta}^{h}\right)$ as $h \rightarrow 0$, as formulated by Shkalikov [15] for $\delta=0$. We give a geometric criterion for $E$ not to belong to $\lim _{h} \operatorname{Sp}\left(H_{\delta}^{h}\right)$ : namely $\lim _{h} \operatorname{Sp}\left(H_{\delta}^{h}\right) \subset \mathcal{T}_{\delta}^{c}, \quad \mathcal{T}_{\delta}^{c}=\mathbb{C} \backslash \mathcal{T}_{\delta}$ where $\mathcal{T}_{\delta}$ is the set of $E \in \mathbb{C}$ such that there exists a progressive path from -1 to 1 with respect to $V_{\delta, \beta}$. ( We define this concept later). This is done in section 3, see Theorem 2. This criterion is not sharp, and also not so easy to use. It is proved using either ellipticity or exact WKB .

As a second step we give an alternate criterion for $E$ to belong to $\lim _{h} \operatorname{Sp}\left(H_{\delta}^{h}\right)$, when $E$ is large enough, which is sharp and computable, see Theorem 3 .

As an application we prove that the perturbation $\delta$ changes the spectrum near $\infty$ quite drastically, see Theorem 7 . The pseudo spectrum is defined to be the limit as $\delta \rightarrow 0$ of the union of spectra over all perturbations of $H^{h}$ of size $\delta$, and we show that this perturbation is sufficient to reach the entire pseudo spectrum of $H^{h}$, see section 7. We note also the work in progress of M. Hager that studies the effect of different perturbations on the spectrum.

We use the geometric criterion for $P^{h}$ to characterize the full set $\mathcal{T}_{0}^{c}$, see Theorem 10, using special properties of the potential, and see that $\mathcal{T}_{0}^{c}$ forms a $Y$ shape. Shkalikov [16] find the same $\lim _{h} \operatorname{Sp}\left(H_{\delta}^{h}\right)$ for two situations, one when $V$ is an one-to-one function on $\mathbb{R}$, the other when $V=i x^{2}$ using a asymptotic similar to exact WKB solution but valid only for this potential, see also [6], [15]. The algorithm proposed by Miller in [13] to study the spectrum is the same as the one we use here, though he applies it to a different operator.

We also obtain a description of the spectrum of $H^{h}$ which is near to $\lim _{h} \operatorname{Sp}\left(P^{h}\right)$ and outside form a compact see Theorem 5 . In the self-adjoint case; this can be found in the book of Marchenko [11] or the paper of Kappeler and Möhr [10], and in some special non self-adjoint cases in work of Carlson, Threadgill and Shubin [1]. However, these results apply for potentials $V$ with $V^{\prime} \in L_{l o c}^{1}$, hence do not pertain to $V_{\delta, \beta}$.

Even though it is not self-adjoint, the operator $H_{\delta, \beta}^{h}$ has discrete spectrum. Indeed, $V_{\delta, \beta}$ is compact relative to $\Delta$, and from Weyl's theorem it follows that its essential spectrum is empty. In particular $H_{\delta, \beta}^{h}$ has no residual spectrum.

2. Generals tools. In this section we are interested in the Dirichlet eigenvalues of the operator $H^{h}$ on $L^{2}([-1,1])$ defined by

$$
\left\{\begin{array}{l}
H^{h} u=E u, \\
u(-1)=u(1)=0 .
\end{array}\right.
$$


Definition 1. A point $x_{0}$ in the complex plane is a turning point of order $k$ for the operator $H^{h}-E$ if $V(x)-E$ vanishes to order $k$ at $x=x_{0}$.

Definition 2. Let $x$ be a point in the complex plane. The Stokes line starting from $x$ is the set

$$
\mathcal{L}(x)=\left\{y \in \mathbb{C}, \operatorname{Re} \int_{x}^{y}(V(t)-E)^{1 / 2} d t=0\right\}
$$

This is independent of the branch of the square root. Denote by $S_{x, y}(E)=$ $\int_{x}^{y}(V(t)-E)^{1 / 2} d t$ for some choice of branch.

Notice that the Stokes lines are integral curves of the Stokes vector field

$$
s(x)=i \overline{(V(x)-E)^{1 / 2}} .
$$

The local structure of Stokes lines can be easily investigated (see e.g. [12], [7]). Away from turning points or singularities of $V$, the Stokes lines form a non singular analytic foliation. Indeed, if $\Omega \subset \mathbb{C}$ does not contain any singularities of $(V-E)^{1 / 2}$, then $x \mapsto z(x, E)=\int_{x_{0}}^{x}(V(t)-E)^{1 / 2} d t$ is an analytic diffeomorphism. Notice also that from a simple turning point $x_{0}$ emanates three Stokes lines, each making an angle of $2 \pi / 3$ with any other at $x_{0}$.

Using ideas from quantum resonance theory, we shall consider as in [7] some distorted operators associated to $H$. Let $\gamma:[-1,1] \ni t \rightarrow \mathbb{C}$ be a smooth simple path in the complex plane $\gamma(-1)=-1$ and $\gamma(1)=1$. Since $V$ is analytic, we can define an operator $H^{\gamma, h}$ on $L^{2}([-1,1])$ as the restriction of $H^{h}$ to $\gamma([-1,1])$. One computes that

$$
H^{\gamma, h}=-h^{2}\left(\frac{1}{\gamma^{\prime}(x)} \frac{d}{d x}\right)\left(\frac{1}{\gamma^{\prime}(x)} \frac{d}{d x}\right)+V(\gamma(x))
$$

The correspondence between the Stokes geometry and spectral properties of $H^{h}$ is given in the following result from [7].

Proposition 1. The operator $H^{\gamma, h}-E$ is elliptic if and only if $\gamma$ is transverse to the Stokes lines.

Proof. The semi-classical principal symbol of $H^{\gamma, h}-E$ is

$$
h^{\gamma}(t, \tau)=\frac{1}{\left(\gamma^{\prime}(t)\right)^{2}}\left(\tau^{2}+\left(\gamma^{\prime}(t)\right)^{2}(V(\gamma(t))-E)\right)
$$

We set $(V(\gamma(t))-E)^{\frac{1}{2}}=r e^{i \theta}$ and $\gamma^{\prime}(t)=e^{i \theta^{\prime}}$ (one can always suppose that $\left|\gamma^{\prime}(t)\right|=$ $1)$. Then the path $\gamma$ is transversal to the Stokes lines if and only if

$$
\operatorname{det}\left(\gamma^{\prime}(t), s(\gamma(t))\right) \neq 0,
$$

where $s(x)$ is the Stokes field defined in (6). This condition is the same as

$$
\operatorname{Re} \gamma^{\prime}(t)\left(V(\gamma(t)-E)^{1 / 2} \neq 0,\right.
$$

or finally $\theta+\theta^{\prime} \not \equiv \pi / 2 \bmod [\pi]$. Since $\operatorname{Im}\left(\gamma^{\prime}(t)^{2} h^{\gamma}\right)=r^{2} \sin 2\left(\theta+\theta^{\prime}\right)$ and $\operatorname{Re}\left(\gamma^{\prime}(t)^{2} h^{\gamma}\right)=\tau^{2}+r^{2} \cos 2\left(\theta+\theta^{\prime}\right)$, the transversality condition is equivalent to the invertibility of $h^{\gamma}$, as stated in the proposition. 
We deduce the following proposition, but this will be improved in Theorem 1 below, where we control the dependence on $E$ and introduce the perturbation.

Proposition 2. For $h$ small enough, $E \notin \mathrm{Sp}\left(H^{h}\right)$ if there exists a path $\gamma$ form -1 to 1 which is transverse to the Stokes lines.

Proof. General properties of distorted analytic operators imply that $E \in \operatorname{Sp}\left(H^{h}\right)$ is equivalent to $E \in \operatorname{Sp}\left(H^{\gamma, h}\right)$. Let $\gamma$ be a path transverse to the Stokes lines. Then by Proposition $1, H^{\gamma, h}-E$ is elliptic and invertible for sufficiently small $h$ (depending on $E$ ).

3. The semi-classical limit of the spectrum. The section is devoted to the proof of the Theorem 1 and a direct application Theorem 2. The next sections 4 and 5 are devoted to the proof of a converse of Theorem 2 .

We now introduce some notation. Denote by $\lim _{h} \operatorname{Sp}\left(H^{h}\right)$ the set of all $E_{0}$ such that there exists a sequence $h_{j} \rightarrow 0$ with $E_{h_{j}} \in \operatorname{Sp}\left(H^{h_{j}}\right)$ and $E_{h_{j}} \rightarrow E_{0}$. For $a \in \mathbb{C}$, we denote by $z_{0}(x)$ and $z_{l, r}(x)$ the action integrals

$$
\begin{gathered}
z_{0}(x)=\int_{\gamma(-1, x)}(V(t)-E)^{1 / 2} d t, z_{l}(x)=\int_{\gamma_{l}(\beta, x)}(V(t)-i \delta-E)^{1 / 2} d t, \\
z_{r}(x)=\int_{\gamma_{r}(\beta, x)}(V(t)+i \delta-E)^{1 / 2} d t
\end{gathered}
$$

where $\gamma(-1, x)$ and $\gamma_{l, r}(\beta, x)$ are paths from -1 or $\beta$ to $x$ on the Riemann surface associated to a choice of $\Sigma_{0}$ of $t \mapsto(V(t)-E)^{1 / 2}$ and $\Sigma_{l, r}$ associated to a choice of $t \mapsto(V(t) \pm i \delta-E)^{1 / 2}, E \in \mathbb{C}$ respectively.

We denote by $\mathcal{T}_{\delta}$ the set of $E \in \mathbb{C}$ such that there exists $\gamma_{l}(-1, \beta)$ on which the function $t \mapsto \operatorname{Re}\left(z_{l}\left(\gamma_{l}(t)\right)\right)$ is strictly monotone, and a path $\gamma_{r}(\beta, 1)$ on which the function $t \mapsto \operatorname{Re}\left(z_{r}\left(\gamma_{r}(t)\right)\right)$ is strictly monotone. We will call such a path 'progressive' . For $\delta=0$ we take a different definition: $E \in \mathcal{T}_{0}$ if and only if there exists a path $\gamma$ from -1 to 1 on which the function $\left.t \mapsto \operatorname{Re}\left(z_{0}(\gamma(t))\right)\right)$ is strictly monotone.

Finally, for $E \in \mathcal{T}_{\delta}$, we denote by

$$
\begin{gathered}
d\left(E, \mathcal{T}_{\delta}^{c}, \gamma\right)=\inf _{l, r} \inf _{x \in \gamma^{l, r}}\left|\operatorname{Re} \partial_{x} z^{l, r}(x)\right| \quad \text { if } \delta \neq 0 \\
d\left(E, \mathcal{T}_{0}^{c}, \gamma\right)=\inf _{x \in \gamma}\left|\operatorname{Re} \partial_{x} z^{0}(x)\right|
\end{gathered}
$$

where $\gamma$ is a progressive path associated to $E\left(\gamma=\cup_{l, r} \gamma^{l, r}\right.$ if $\left.\delta \neq 0\right)$.

REMARK 1. Notice that $\mathcal{T}_{\delta}$ is open. More precisely, if $c$ is small enough there exists $C>0$, such that if $E_{0}$ belongs to $\mathcal{T}_{\delta}$ then, for $E$ such that $\left|E-E_{0}\right| \leq c d\left(E_{0}, \mathcal{T}_{\delta}^{c}, \gamma\right)$, we get $E \in \mathcal{T}_{\delta}$ and $d\left(E, \mathcal{T}_{\delta}^{c}, \gamma\right) \geq C d\left(E_{0}, \mathcal{T}_{\delta}^{c}, \gamma\right)$. This follows from the relation

$$
\sqrt{V_{\delta, \beta}-E}=\sqrt{V_{\delta, \beta}-E_{0}}+O\left(\frac{E-E_{0}}{\sqrt{V_{\delta, \beta}-E_{0}}}\right)
$$

which shows that a progressive path for $E_{0}$ is also a progressive path for $E$.

TheOrem 1. Let $\delta \geq 0$ be small enough and $E \in \mathcal{T}_{\delta}$. Then $E \notin \operatorname{Sp}\left(H_{\delta}^{h}\right)$ as soon as $h \leq d\left(E, \mathcal{T}_{\delta}^{c}, \gamma\right)^{7}$. (The reason for the exponent 7 will emerge at the end of the proof.) 
From Remark 1 and Theorem 1 we obtain the

THEOREM 2. If $E_{0} \in \lim _{h} \operatorname{Sp}\left(H_{\delta}^{h}\right)$, then $E_{0} \in \mathcal{T}_{\delta}^{c}$.

Proof. Suppose that $E_{0} \in \mathcal{T}_{\delta}$ and $\gamma$ is a progressive path associated to $E_{0}$. Let $E_{h}$ be a sequence in the spectrum of $H_{\delta}^{h}$ tending to $E_{0}$. By the remark above, $E_{h}$ belongs to $\mathcal{T}_{\delta}$ as soon as $h$ is small enough, and we get $d\left(E_{h}, \mathcal{T}_{\delta}^{c}, \gamma\right) \geq \tilde{C}_{0}$. Theorem 1 shows that $E_{h}$ is not in the spectrum of $H_{\delta}^{h}$ as soon as $h$ is small. This contradicts the hypothesis, so $E_{0} \in \mathcal{T}_{\delta}^{c}$.

Proof of Theorem 1. We denote by $\left(u_{+}^{l}, u_{-}^{l}\right)$ a basis of the space of solutions of the equation

$$
\left(H_{\delta}^{h}-E\right) u=0
$$

in $L^{2}([-1, \beta])$, and by $\left(u_{+}^{r}, u_{-}^{r}\right)$ a basis of the space of solutions of $(10)$ in $L^{2}([\beta, 1])$. A function $f \in L^{2}([-1,1])$ is an eigenfunction of $H_{\delta}$ with eigenvalue $E \in \mathbb{C}$, if and only if $f$ solves the equation in each subinterval, $f(1)=f(-1)=0$, and both $f$ and $\partial_{x} f$ are continuous at $x=\beta$. Writing

$$
f=\alpha_{+}^{l} u_{+}^{l}+\alpha_{-}^{l} u_{-}^{l} \text { on }[-1, \beta] \quad f=\alpha_{+}^{r} u_{+}^{r}+\alpha_{-}^{r} u_{-}^{r} \text { on }[\beta, 1],
$$

Then $f$ is an eigenfunction of $H_{\delta}^{h}$ with eigenvalue $E \in \mathbb{C}$ if and only if

$$
\left(\begin{array}{cccc}
u_{-}^{l}(-1) & u_{+}^{l}(-1) & 0 & 0 \\
u_{-}^{l}(\beta) & u_{+}^{l}(\beta) & -u_{-}^{r}(\beta) & -u_{+}^{r}(\beta) \\
\partial_{x} u_{-}^{l}(\beta) & \partial_{x} u_{+}^{l}(\beta) & -\partial_{x} u_{-}^{r}(\beta) & -\partial_{x} u_{+}^{r}(\beta) \\
0 & 0 & u_{-}^{r}(1) & u_{+}^{r}(1)
\end{array}\right)\left(\begin{array}{c}
\alpha_{+}^{l} \\
\alpha_{-}^{l} \\
\alpha_{+}^{r} \\
\alpha_{-}^{r}
\end{array}\right)=0 .
$$

Therefore $E$ belongs to $\operatorname{Sp}\left(H_{\delta}^{h}\right)$, the spectrum of $H_{\delta}^{h}$, if and only if

$$
\operatorname{det} \mathcal{I}(\beta, E)=0
$$

where $\mathcal{I}(\beta, E)$ is the matrix appearing in (12).

REMARK 2. For $\delta=0$, the proof is slightly different, the corresponding matrix is

$$
\mathcal{I}(\beta, E)=\left(\begin{array}{cc}
u_{-}(-1) & u_{+}(-1) \\
u_{-}(1) & u_{+}(1)
\end{array}\right),
$$

We leave details to the reader and in the following, treat only the case $\delta \neq 0$.

In the sequel, we shall compute this determinant for the two particular bases $\left(u_{+}^{l}, u_{-}^{l}\right)$ and $\left(u_{+}^{r}, u_{-}^{r}\right)$, namely for complex WKB solutions as in [8], or [7]. Suppose $E \in T_{\delta}$. Then there is a path $\gamma_{l}=\gamma_{l}(-1, \beta)$ transversal to the Stokes lines $\mathcal{L}(y)$, and we can suppose that $\gamma_{l}(-1, \beta)$ is of type + , that is $t \mapsto z_{l}\left(\gamma_{l}(t)\right)$ is strictly increasing. Indeed if it is not the case, the path on the other sheet of the Riemann surface $\Sigma_{l}$ associated to the definition of the square root with the same projection on $\mathbb{C}$ as $\gamma_{l}(-1, \beta)$ is then of type + . (We have $z_{l}(-1)<0$.) We define two independent complex WKB solutions $w_{ \pm}$of the equation $\left(H_{\delta}^{h}-E\right) u=0$ on the interval $[-1, \beta]$.These have the form

$$
\begin{aligned}
w_{ \pm}^{l}: x \mapsto & (V(x)-i \delta-E)^{-\frac{1}{4}} e^{ \pm z_{l}(x) / h} W_{ \pm}^{l}\left(z_{l}(x)\right) \\
= & \left(\partial_{x} z_{l}(x)\right)^{-\frac{1}{2}}(x) e^{ \pm z_{l}(x) / h} W_{ \pm}^{l}\left(z_{l}(x)\right)
\end{aligned}
$$


Similarly, there exists a path $\gamma_{r}=\gamma_{r}(\beta, 1)$ transverse to the Stokes lines of type + , (We have $z_{r}(1)>0$ and we have again two independent complex WKB solutions of the equation $H_{\delta} w_{ \pm}=0$ on the interval $[\beta, 1]$ :

$$
\begin{array}{r}
w_{ \pm}^{r}: x \mapsto(V(x)+i \delta-E)^{-\frac{1}{4}} e^{ \pm z_{r}(x) / h} W_{ \pm}^{l}\left(z_{r}(x)\right) \\
=\left(\partial_{x} z_{r}(x)\right)^{-\frac{1}{2}} e^{ \pm z_{r}(x) / h} W_{ \pm}^{r}\left(z_{r}(x)\right) .
\end{array}
$$

The functions $W_{ \pm}^{l, r}$ are convergent series of the form

$$
W_{ \pm}^{l, r}=\sum_{n=0}^{\infty} W_{n, \pm}^{l, r},
$$

where the $W_{n, \pm}^{l, r}$ are determined by the recurrence relations:

$$
\left\{\begin{array}{l}
\left(\partial_{z} \pm \frac{2}{h}\right) W_{2 n+1, \pm}^{l, r}=-H^{l, r} W_{2 n, \pm}^{l, r} \\
\partial_{z} W_{2 n, \pm}^{l, r}=-H^{l, r} W_{2 n-1, \pm}^{l, r}
\end{array}\right.
$$

with initial data

$$
\begin{cases}W_{0, \pm}^{l, r}=1 \\ W_{n,+}^{l}\left(z_{l}(-1)\right)=0, W_{n,-}^{r}\left(z_{r}(1)\right)=0, & n \geq 1, \\ W_{n,-}^{l}\left(z_{l}(\beta)\right)=0, W_{n,+}^{r}\left(z_{r}(\beta)\right)=0, & n \geq 1,\end{cases}
$$

We have set here

$$
H^{l, r}\left(z_{l, r}(x)\right)=-\frac{1}{4} \frac{\partial_{x} V(x)}{\left(\partial_{x} z_{l, r}(x)\right)^{3}} .
$$

These equations can be written in integral form as

$$
\left\{\begin{array}{c}
W_{2 n+1, \pm}^{r, l}=I_{ \pm}^{l, r}\left(W_{2 n, \pm}^{r, l}\right), \\
W_{2 n+2, \pm}^{r, l}=J^{l, r}\left(W_{2 n+1, \pm}^{r, l}\right)
\end{array}\right.
$$

where

$$
\left\{\begin{array}{l}
I_{ \pm}^{l, r}(v)(z)=-\int_{\tilde{\gamma}_{l, r}(z)} e^{ \pm 2(u-z) / h} H^{l, r}(u) v(u) d u \\
J^{l, r}(v)(z)=-\int_{\tilde{\gamma}_{l, r}(z)} H^{l, r}(u) v(u) d u
\end{array}\right.
$$

and $\tilde{\gamma}_{l, r}(z)$ is the image by $x \mapsto z_{l, r}(x)$ of $\gamma_{l, r}$ from the initial point -1 to $z$ for $l$ and from $\beta$ to $z$ for $r$.

Now we have the following estimates

$$
\begin{aligned}
\sup _{z \in \tilde{\gamma}_{l, r}}\left|I_{+}^{l, r}(v)(z)\right| & \leq \sup _{z \in \tilde{\gamma}_{l, r}}|v(z)| \sup _{z \in \tilde{\gamma}_{l, r}, z=z^{l, r}(x)}\left\{\left|H^{l, r}(z)\right| \frac{\left|\partial_{x} z_{l, r}(x)\right|}{\left|\operatorname{Re} \partial_{x} z_{l, r}(x)\right|}\right\} \times \\
& \left|\int_{\tilde{\gamma}_{l, r}} e^{\operatorname{Re}\left(\frac{2(u-z)}{h}\right)} \operatorname{Re}(d z)\right| \\
& \leq h \sup _{z \in \tilde{\gamma}_{l, r}}|v(z)| \sup _{z \in \tilde{\gamma}_{l, r}, z=z^{l, r}(x)}\left|H^{l, r}(z)\right|\left|\partial_{x} z_{l, r}(x)\right| \frac{1}{d\left(E, \mathcal{T}_{\delta}^{c}, \gamma\right)} \\
& \leq C(V) h \sup _{z \in \tilde{\gamma}_{l, r}}|v(z)| \frac{1}{d\left(E, \mathcal{T}_{\delta}^{c}, \gamma\right)^{3}}
\end{aligned}
$$




$$
\begin{aligned}
\sup _{z \in \tilde{\gamma}_{l, r}}\left|J^{l, r}(v)(z)\right| & \leq \sup _{z \in \tilde{\gamma}_{l, r}}|v(z)| \sup _{z \in \tilde{\gamma}_{l, r}, z=z^{l, r}(x)}\left|H^{l, r}(z)\right| \partial_{x} z_{l, r}(x) \mid \\
& \leq \sup _{z \in \tilde{\gamma}_{l, r}}|v(z)| \sup _{z \in \tilde{\gamma}_{l, r}, z=z^{l, r}(x)}\left|H^{l, r}(z)\right|\left|\partial_{x} z_{l, r}(x)\right| \\
& \leq C(V) \sup _{z \in \tilde{\gamma}_{l, r}}|v(z)| \frac{1}{d\left(E, \mathcal{T}_{\delta}^{c}, \gamma\right)^{2}} .
\end{aligned}
$$

Similarly we obtain

$$
\begin{aligned}
\sup _{z \in \tilde{\gamma}_{l, r}}\left|I_{-}^{l, r}(v)(z)\right| & \leq h \sup _{z \in \tilde{\gamma}_{l, r}}|v(z)| \sup _{z \in \tilde{\gamma}_{l, r}, z=z^{l, r}(x)}\left|H^{l, r}(z)\right|\left|\partial_{x} z_{l, r}(x)\right| \frac{1}{d\left(E, \mathcal{T}_{\delta}^{c}, \gamma\right)} \\
& \leq C(V) h \sup _{z \in \tilde{\gamma}_{l, r}}|v(z)| \frac{1}{d\left(E, \mathcal{T}_{\delta}^{c}, \gamma\right)^{3}}
\end{aligned}
$$

We denote by $\lfloor x\rfloor$ the integer part of $x$. Hence on a progressive path $\gamma$ we have

$$
\left|W_{n, \pm}^{l, r}\right|_{\infty} \leq \frac{h^{\left\lfloor\frac{n+1}{2}\right\rfloor}}{d\left(E, \mathcal{T}_{\delta}^{c}, \gamma\right)^{3 n}}
$$

which means that

$$
\begin{aligned}
& W_{+}^{l}(-1)=1, \quad W_{+}^{l}(\beta)=1+O\left(\frac{h}{d\left(E, \mathcal{T}_{\delta}^{c}, \gamma\right)^{6}}\right), \\
& W_{-}^{l}(\beta)=1, \quad W_{-}^{l}(-1)=1+O\left(\frac{h}{d\left(E, \mathcal{T}_{\delta}^{c}, \gamma\right)^{6}}\right), \\
& W_{+}^{r}(\beta)=1, \quad W_{+}^{r}(1)=1+O\left(\frac{h}{d\left(E, \mathcal{T}_{\delta}^{c}, \gamma\right)^{6}}\right), \\
& W_{-}^{r}(1)=1, \quad W_{-}^{r}(\beta)=1+O\left(\frac{h}{d\left(E, \mathcal{T}_{\delta}^{c}, \gamma\right)^{6}}\right) .
\end{aligned}
$$

The function $w_{ \pm}^{l}$ form a basis of solutions of the equation $H_{\delta} w=0$, and similarly $w_{ \pm}^{r}$ is a basis of solutions of the equation $H_{\delta} w=0$. A computation gives

$$
\frac{d w_{ \pm}^{l, r}}{d x}(x)= \pm \partial_{x} z_{l, r}(x)^{\frac{1}{2}} e^{ \pm \frac{z_{l, r}(x)}{h}} \frac{1}{h} \sum_{n=0}^{\infty}(-1)^{n} W_{n}^{l, r}\left(z_{l, r}(x)\right),
$$

and so

$$
\frac{d w_{ \pm}^{l, r}}{d x}(x)= \pm \partial_{x} z_{l, r}(x)^{\frac{1}{2}} e^{ \pm \frac{z_{l, r}(x)}{h}} \frac{1}{h}\left(1+O\left(\frac{h}{d\left(E, \mathcal{T}_{\delta}^{c}, \gamma\right)^{6}}\right)\right) .
$$

Now compute the determinant; we shall write $\mathcal{O}=\mathcal{O}\left(\frac{h}{d\left(E, \mathcal{T}_{\delta}^{c}, \gamma\right)^{6}}\right)$ for simplicity.

$$
\begin{aligned}
& \operatorname{det}\left(\begin{array}{llll}
w_{-}^{l}(-1) & w_{+}^{l}(-1) & 0 & 0 \\
w_{-}^{l}(\beta) & w_{+}^{l}(\beta) & -w_{-}^{r}(\beta) & \left.-w_{+}^{r}(\beta)\right) \\
w_{-}^{l^{\prime}}(\beta) & w^{l^{\prime}}(\beta) & -w_{-}^{r \prime}(\beta) & -w_{+}^{r \prime}(\beta) \\
0 & 0 & w_{-}^{r}(1) & w_{+}^{r}(1)
\end{array}\right)= \\
& \frac{1}{h} \partial_{x} z_{r}^{-\frac{1}{2}}(1) \partial_{x} z_{r}^{-\frac{1}{2}}(\beta) \partial_{x} z_{l}^{-\frac{1}{2}}(\beta) \partial_{x} z_{l}^{-\frac{1}{2}}(-1) e^{-\frac{z_{l}(-1)}{h}} e^{\frac{z_{r}(1)}{h}} \times \\
& \left|\begin{array}{llll}
1+\mathcal{O} & e^{2 \frac{z_{l}(-1)}{h}} & 0 & 0 \\
1 & 1+\mathcal{O} & -1+\mathcal{O} & -1 \\
-\partial_{x} z_{l}(\beta)(1+\mathcal{O}) & \partial_{x} z_{l}(\beta)(1+\mathcal{O}) & \partial_{x} z_{r}(\beta)(1+\mathcal{O}) & -\partial_{x} z_{r}(\beta)(1+\mathcal{O}) \\
0 & 0 & e^{-\frac{z_{r}(1)}{h}} & 1+\mathcal{O}
\end{array}\right|
\end{aligned}
$$


So the sign of the determinant is given by the sign of

$$
\begin{aligned}
& \partial_{x} z_{l}^{-\frac{1}{2}}(-1) \partial_{x} z_{r}^{-\frac{1}{2}}(\beta) \partial_{x} z_{l}^{-\frac{1}{2}}(\beta) \partial_{x} z_{r}^{-\frac{1}{2}}(1) \times \\
& \quad\left(\operatorname{det}\left(\begin{array}{llll}
1 & 0 & 0 & 0 \\
0 & 1 & -1 & 0 \\
0 & \partial_{x} z_{l}(\beta) & \partial_{x} z_{r}(\beta) & 0 \\
0 & 0 & 0 & 1
\end{array}\right)+\mathcal{O}\left(\frac{h}{d\left(E, \mathcal{T}_{\delta}^{c}, \gamma\right)^{6}}\right)\right) \\
& =\partial_{x} z_{l}^{-\frac{1}{2}}(-1) \partial_{x} z_{r}^{-\frac{1}{2}}(\beta) \partial_{x} z_{l}^{-\frac{1}{2}}(\beta) \partial_{x} z_{r}^{-\frac{1}{2}}(1) \times \\
& \left(\partial_{x} z_{r}(\beta)+\partial_{x} z_{l}(\beta)+\mathcal{O}\left(\frac{h}{d\left(E, \mathcal{I}_{\delta}^{c}, \gamma\right)^{6}}\right)\right) \\
& =(V(-1)-i \delta-E)^{-\frac{1}{4}}(V(\beta)-i \delta-E)^{-\frac{1}{4}} \\
& \times(V(\beta)+i \delta-E)^{-\frac{1}{4}}(V(1)+i \delta-E)^{-\frac{1}{4}} \\
& \times\left(\sqrt{V(\beta)+i \delta-E}+\sqrt{V(\beta)-i \delta-E}+\mathcal{O}\left(\frac{h}{d\left(E, \mathcal{T}_{\delta}^{c}, \gamma\right)^{6}}\right)\right)
\end{aligned}
$$

( we have $\operatorname{Re}(\sqrt{V(\beta)+i \delta-E}+\sqrt{V(\beta)-i \delta-E}) \geq 2 d\left(E, \mathcal{T}_{\delta}^{c}, \gamma\right)$.)

The determinant is non zero as soon as $h$ is small compared to $d\left(E, \mathcal{T}_{\delta}^{c}, \gamma\right)^{7}$.

4. spectrum for large $E$ and $\delta=0$. In this section we prove three results: the first, Theorem 3, gives the condition for $E_{0}$ to belong to $\lim _{h} \operatorname{Sp}\left(H^{h}\right)$. The second, Theorem 4, describes $\lim _{h} \operatorname{Sp}\left(H^{h}\right)$ as a curve and gives its asymptotics near $\infty$. The last, Theorem 5, describes the eigenvalues of $H^{h}$ which are near to $\lim _{h} \operatorname{Sp}\left(H^{h}\right)$. In the following we let denote by $Y$ a primitive of the potential $V$. In this section, we assume that $V$ verifies the following hypothesis: (H1) For any $\Omega \subset \mathbb{C}$, if $V^{-1}(\Omega)$ bounded then $\Omega$ is relatively compact.

(H2) If $E$ is large enough,but with small imaginary part, then 1 and -1 belong to the same Stokes region. We recall that this means that one can find a path going from -1 to 1 that does not intersect the Stokes lines issuing from the turning points. Notice that, under the assumption (H2), $E \in \mathcal{T}_{0}^{c}$ is equivalent to $\operatorname{Re}\left(\int_{-1}^{1} \sqrt{V-E} d x\right)=0$.

THEOREM 3. For $E_{0}$ large with small enough imaginary part, then $\operatorname{Re}\left(\int_{-1}^{1} \sqrt{V-E_{0}} d x\right)=0$ if and only if $E_{0} \in \lim _{h} \operatorname{Sp}\left(H_{0}^{h}\right)$.

Moreover there exists $C$ small enough and $E_{h}$ in the spectrum of $H_{0}^{h}$ which satisfies $\left|\sqrt{E_{h}}-\sqrt{E_{0}}\right| \leq C h$.

Proof of Theorem 3. The reverse implication follows directly from Theorem 2, so we prove the direct implication.

There exists two WKB solutions $w_{ \pm}$of $\left(H_{0}^{h}-E\right) w=0$ of the form

$$
\begin{gathered}
w^{ \pm}(x)=\partial_{x} z_{0}(x)^{-\frac{1}{2}} e^{ \pm z_{0}(x) / h} W_{ \pm}\left(z_{0}(x)\right), \\
W_{ \pm}=\sum_{j=0} W_{n, \pm} \quad W_{0, \pm}(-1)=1, \quad W_{n, \pm}(-1)=0 \text { for } n>0 .
\end{gathered}
$$

The complex number $E$ belongs to the spectrum of $H_{0}^{h}$ if and only if

$e^{\frac{2}{h} z_{0}(1)}=\frac{W_{+}(1)}{W_{-}(1)}$, which is equivalent to the existence of $k \in \mathbb{Z}$ such that

$$
\int_{-1}^{1} \sqrt{V(x)-E} d x-\frac{h}{2} \ln \left(W_{+}(1)\right)+\frac{h}{2} \ln \left(W_{-}(1)\right)-i h k \pi=0 .
$$


Let us denote

$$
f(E)=\int_{-1}^{1} \sqrt{V(x)-E} d x-\operatorname{Im} \int_{-1}^{1} \sqrt{V(x)-E_{0}} d x
$$

and

$$
k_{0}=\left\lfloor\frac{\operatorname{Im} \int_{-1}^{1} \sqrt{V(x)-E_{0}} d x}{h}\right\rfloor
$$

and also

$$
g(E)=\int_{-1}^{1} \sqrt{V(x)-E} d x-\frac{h}{2} \ln \left(W_{+}(1)\right)+\frac{h}{2} \ln \left(W_{-}(1)\right)-i h k_{0} \pi .
$$

We have $f\left(E_{0}\right)=0$. We want to apply Rouché's theorem, but to do so we must give upper and lower bounds for $W_{ \pm}(1)$ and an estimate on $f^{\prime}\left(E_{0}\right)=-\frac{1}{2} \int_{-1}^{1} \frac{1}{\sqrt{V(x)-E_{0}}} d x$.

By the hypothesis on the geometry of the Stokes lines near -1 and 1 there exists a path $\gamma$ which links -1 to 1 on which $\operatorname{Re} \int_{-1}^{t} \sqrt{V(x)-E_{0}} d x=0$ for all $t \in \gamma$. Moreover $V(\gamma)$ is bounded uniformly with respect to $E_{0}$, and by assumption (H1), $\gamma$ is also bounded uniformly with respect to $E_{0}$.

We have

$$
\frac{1}{\sqrt{V(x)-E_{0}}}=\frac{1}{i \sqrt{E}_{0}}\left(1+\mathcal{O}\left(\frac{\sup _{\gamma} V}{E_{0}}\right)\right)
$$

therefore

$$
\begin{gathered}
\left|f(E)-f\left(E_{0}\right)\right| \geq \frac{1}{2 \sqrt{\left|E_{0}\right|}}\left|\left(E-E_{0}\right)\right|, \\
\sup _{\gamma}|H(z(x))| \leq C_{1} \frac{\sup _{\gamma}\left|V^{\prime}(x)\right|}{\left|E_{0}\right|^{\frac{3}{2}}},
\end{gathered}
$$

and

$$
\sup _{b, b^{\prime} \in \gamma}\left|\operatorname{Re} \int_{b}^{b^{\prime}} \sqrt{V(t)-E} d t\right| \leq C_{2} \frac{E-E_{0}}{\sqrt{\left|E_{0}\right|}}
$$

for any $E$ and $\alpha_{0}$ such that $\left|E-E_{0}\right| \leq \mathrm{o}\left(\mathrm{E}_{0}\right)$ and $\left|E_{0}\right|>C_{0}$. Using the expression of $W_{n,+}(z)$ as a Volterra integral, we obtain the estimates

$$
\left|W_{n, \pm}(z)\right| \leq \exp \left\{\frac{2}{h} \sup _{b, b^{\prime} \in \gamma}\left|\operatorname{Re} \int_{b}^{b^{\prime}} \sqrt{V(t)-E} d t\right|(n+2)\right\} \sup _{\gamma}|H|^{n} \frac{1}{n !} .
$$

This gives

$$
\left|W_{n, \pm}(z)\right| \leq \exp \left\{\frac{C_{2}}{h} \frac{\left|E-E_{0}\right|}{\sqrt{\left|E_{0}\right|}} n+2\right\} \frac{\hat{C}_{1}^{n}}{\left|E_{0}\right|^{\frac{3 n}{2}} n !}
$$

for $\left|E-E_{0}\right| \leq h_{0}\left(E_{0}\right)$ and $\left|E_{0}\right|>C_{0}$. So we obtain

$$
W_{ \pm}(1)=1+\frac{\hat{C}_{1}}{\left|E_{0}\right|^{\frac{3}{2}}} \exp \left\{\frac{2 C_{2}}{h} \frac{\left|E-E_{0}\right|}{\sqrt{\left|E_{0}\right|}}\right\} \exp \left\{\frac{\hat{C}_{1}}{\left|E_{0}\right|^{\frac{3}{2}}} e^{\frac{2 C_{2}}{h}} \frac{\left|E-E_{0}\right|}{\sqrt{\left|E_{0}\right|}}\right\}
$$


This gives the estimate

$$
\left|W_{ \pm}(1)-1\right| \leq \frac{\tilde{C}_{1}}{\left|E_{0}\right|^{\frac{3}{2}}} e^{C_{2} C_{3}} e^{\hat{C}_{1}\left|E_{0}\right|^{-\frac{3}{2}} e^{C_{2} C_{3}}}
$$

for $E_{0}>C_{0}$ and $\left|E-E_{0}\right| \leq C_{3} h \sqrt{\left|E_{0}\right|}$ with $h$ small enough. We also have

$$
\left|\ln W_{+}(1)\right|+\left|\ln W_{-}(1)\right| \leq \frac{\check{C}_{1}}{\left|E_{0}\right|^{\frac{3}{2}}}
$$

for $E_{0}>C\left(C_{3}\right)$, and $\left|E-E_{0}\right| \leq C_{3} h \sqrt{\left|E_{0}\right|}$ with $h$ enough small, and for the functions $f$ and $g$ we get

$$
|f(E)-g(E)| \leq h+\frac{h}{2}\left(\left|\ln W_{+}(1)\right|+\left|\ln W_{-}(1)\right|\right) .
$$

Thus if $\left|E-E_{0}\right|=C_{3} h \sqrt{\left|E_{0}\right|}$, for some large constant $C_{3}$, we have

$$
|f(E)-g(E)| \leq\left(1+C_{4}\left|E_{0}\right|^{-\frac{3}{2}}\right) h, \quad|f(E)| \geq \frac{C_{3}}{2} h .
$$

Finally, if $E_{0}$ is large enough, all the assumptions of Rouché's theorem are fulfilled, and we get the result. $\square$

TheOREM 4. For any fixed a large enough, the equation

$$
\operatorname{Re} S_{-1,1}(E)_{\left.\right|_{E=a+i b}}=\operatorname{Re} \int_{-1}^{1} \sqrt{V(x)-(a+i b)} d x=0
$$

has a unique solution $b(a)$. Moreover $b(a)=\frac{1}{2} \operatorname{Im}(Y(1)-Y(-1))+\mathcal{O}\left(\frac{1}{a}\right)$.

Proof. First we prove that if $b$ is such that Re $\int_{-1}^{1} \sqrt{V(x)-a-i b} d x=0$, and $b=\mathrm{o}(\mathrm{a})$ then

$$
b=i \frac{1}{2} \operatorname{Im}(Y(1)-Y(-1))+\mathcal{O}\left(a^{-1}\right) .
$$

Indeed if we denote by $E=a+i b$

$$
\varphi(E, \alpha, y)=\int_{\alpha}^{y} \sqrt{V(x)-E} d x,
$$

then, uniformly for $y$ in a compact set,

$$
\varphi(E, \alpha, y)=i \sqrt{E}(y-\alpha)-i \frac{1}{2 \sqrt{E}}(W(y)-W(\alpha))+O\left(E^{-\frac{3}{2}}\right) .
$$

Since $\operatorname{Re} S_{-1,1}(E)=0$, we have

$$
\operatorname{Re}\left(i 2 \sqrt{E}-i \frac{1}{2 \sqrt{E}}(Y(1)-Y(-1))\right)=\mathcal{O}\left(E^{-\frac{3}{2}}\right) .
$$

Writing $\sqrt{E}=c+i \tilde{b}$, then $\tilde{b}=\mathrm{o}(\mathrm{c})$ and

$$
-2 \tilde{b}+\frac{1}{2 c} \operatorname{Im}(Y(1)-Y(-1))+O\left(\frac{\tilde{b}}{c^{2}}\right)=\mathcal{O}\left(c^{-3}\right),
$$


or equivalently

$$
\tilde{b}=\frac{1}{4 c} \operatorname{Im}(Y(1)-Y(-1))+o\left(\frac{1}{c}\right) .
$$

This gives $b=\frac{1}{2} \operatorname{Im}(Y(1)-Y(-1))+o(1)$, and $b=O(1)$. Therefore $\tilde{b}=O\left(\frac{1}{c}\right)$ and recycling through this argument,

$$
\tilde{b}=\frac{1}{4 c} \operatorname{Im}(Y(1)-Y(-1))+O\left(\frac{1}{c^{3}}\right)
$$

which gives (32).

The existence part of the theorem is straightforward: set

$$
\varphi(c, \hat{b}):=\operatorname{Re} \int_{-1}^{1} \sqrt{c V(x)-1-i \hat{b}} d x=c \operatorname{Re} \int_{-1}^{1} \sqrt{V(x)-E} d x
$$

where $E=\frac{1}{c}(1+i \hat{b})$. Since $\partial_{b} \varphi(0,0)=-2$ and $\varphi(0,0)=0$, the implicit function theorem applies.

Finally, the uniqueness follows from the fact that the map $\phi: b \mapsto$ $\operatorname{Re} \int_{-1}^{1} \sqrt{V(x)-a-i b} d x$ is injective for $a$ large enough. Indeed

$$
\left|\sqrt{V(x)-a-i b}-\sqrt{V(x)-a-i b^{\prime}}\right| \leq C \sqrt{\left|b-b^{\prime}\right|},
$$

for a suitable branch of the square root.

REMARK 3. We can compute more terms of the asymptotic expansion than in (32). Indeed, from

$$
\begin{array}{r}
\varphi(E,-1,1)=i 2 \sqrt{E}-i \frac{1}{2 \sqrt{E}}(Y(1)-Y(-1))+i E^{-\frac{3}{2}} \int_{-1}^{1} V^{2}(x) d x \\
-i E^{-\frac{5}{2}} \int_{-1}^{1} V^{3}(x) d x+O\left(E^{-\frac{7}{2}}\right) .
\end{array}
$$

we get

$$
b(a)=\frac{1}{2} \operatorname{Im}(Y(1)-Y(-1))+\frac{3}{8 a^{2}} \operatorname{Im}\left(\int_{-1}^{1} V^{3}\right)+\mathcal{O}\left(\frac{1}{a^{3}}\right) .
$$

Looking more carefully at the quantization rules, we obtain the asymptotics of the eigenvalues.

THEOREM 5. Let $E_{0}$ be a solution of $\operatorname{Re}\left(\int_{-1}^{1} \sqrt{V-E_{0}} d x\right)=0$ with $\left|E_{0}\right|$ large and $\operatorname{Im}\left(E_{0}\right)$ small. There exist $C>0$ such that if $E_{h}$ is in the spectrum of $H_{0}^{h}$ and satisfies $\left|\sqrt{E_{h}}-\sqrt{E_{0}}\right| \leq C h$ then $E_{h}$ satisfies:

$$
E_{h}=\left(\frac{\pi h k}{2}\right)^{2}+\frac{(Y(1)-Y(-1))}{2}+\frac{(Y(1)-Y(-1))^{2}}{(2 h k \pi)^{2}}+\mathcal{O}\left(\frac{1}{(h k)^{3}}\right) .
$$

for some $k \in \mathbb{N}$. 
Remark 4. For a real potential $V$ bounded on $[-1,1]$ and for any $E_{0} \in \mathbb{R}$ large we get $\operatorname{Re}\left(\int_{-1}^{1} \sqrt{V-E_{0}} d x\right)=0$. So Theorem 5 can be read as: If $E_{h}$ is large and satisfies $E_{h} \in \operatorname{Sp}\left(H_{0}^{h}\right)$ then $E_{h}$ satisfies:

$$
E_{h}=\left(\frac{\pi h k}{2}\right)^{2}+\frac{(Y(1)-Y(-1))}{2}+\frac{(Y(1)-Y(-1))^{2}}{(2 h k \pi)^{2}}+\mathcal{O}\left(\frac{1}{(h k)^{3}}\right) .
$$

for some $k \in \mathbb{N}$.

Proof.

$$
\int_{-1}^{1} \sqrt{V-E} d x=i 2 \sqrt{E}-i \frac{1}{2 \sqrt{E}}(Y(1)-Y(-1))+\mathcal{O}\left(E^{-\frac{3}{2}}\right) .
$$

Then $E \in \operatorname{Sp}\left(H_{\delta}^{h}\right)$ if and only if there exists $k \in \mathbb{Z}$ such that

$$
\int_{-1}^{1} \sqrt{V(x)-E} d x-\frac{h}{2} \ln \left(W_{+}(1)\right)+\frac{h}{2} \ln \left(W_{-}(1)\right)-i h k \pi=0
$$

For $E_{0}$ big enough, $\left|E-E_{0}\right| \leq C_{3} h \sqrt{E_{0}}, C_{3}$ is as in (31) and $h$ small, there exist $\check{C}$ such that

$$
\left|\ln W_{+}(1)\right|+\left|\ln W_{-}(1)\right| \leq \check{C}\left|E_{0}\right|^{-\frac{3}{2}}
$$

So $E \in \operatorname{Sp}\left(H_{\delta}^{h}\right)$ if and only if there exist $k \in \mathbb{Z}$ such that

$$
i 2 \sqrt{E}-i \frac{1}{2 \sqrt{E}}(Y(1)-Y(-1))-i h k \pi=\mathcal{O}\left(E^{-\frac{3}{2}}\right)
$$

In particular taking $\sqrt{E}=c+i \tilde{b}$ the real and imaginary parts give

$$
\begin{gathered}
2 c-h k \pi=\mathcal{O}\left(c^{-1}\right) \quad c=\frac{1}{2} h k \pi+\mathcal{O}\left((k h)^{-1}\right) \\
2 \tilde{b}=\frac{1}{2 c} \operatorname{Im}(Y(1)-Y(-1))+\mathcal{O}\left(c^{-3}\right)
\end{gathered}
$$

Using this again gives

$$
c=\frac{1}{2} h k \pi+\frac{1}{2 h k \pi} \operatorname{Re}(Y(1)-Y(-1))+\mathcal{O}\left((k h)^{-3}\right)
$$

口

Remark 5. One can also treat the case $P^{h}$ on all of $\mathbb{R}$. Then a condition for $E$ to belong to $\lim _{h} \mathrm{Sp}\left(H^{h}\right)$ is that there exists a progressive path joining two turning points. 
5. Large spectrum of a perturbation of $\mathbf{H}$. In this section, we prove two theorems: Theorem 6 gives the condition for $E_{0}$ to belong to $\lim _{h} \operatorname{Sp}\left(H_{\delta}^{h}\right)$ and Theorem 7 gives a description of $\lim _{h} \operatorname{Sp}\left(H_{\delta}^{h}\right)$ as the union of two curves, the asymptotics near $\infty$ of which are made explicit.

We assume that $V$ verifies the hypothesis (H1) and in addition :

(H3) If $E$ is large enough, with small imaginary part then $1,-1$ and $\beta$ belong to the same Stokes region.

We assume $\beta \neq \pm 1$. Recall that (H3) means that one can find paths going from -1 to $\beta$ and from 1 to $\beta$ which do not intersect the Stokes lines issuing from the turning points. With the hypothesis (H3), the condition $E \in \mathcal{T}_{\delta}^{c}$ is then equivalent

to

either $\operatorname{Re}\left(\int_{-1}^{\beta} \sqrt{V-i \delta-E} d x\right)=0$ or $\operatorname{Re}\left(\int_{\beta}^{1} \sqrt{V+i \delta-E} d x\right)=0$.

THEOREM 6. For $E_{0}$ large enough, with small enough imaginary part, then $E_{0}$ satisfies $\operatorname{Re}\left(\int_{-1}^{\beta} \sqrt{V-E_{0}} d x\right)=0$ or $\operatorname{Re}\left(\int_{\beta}^{1} \sqrt{V-E_{0}} d x\right)=0$ if and only if $E_{0} \in$ $\lim _{h} \operatorname{Sp}\left(H_{\delta}^{h}\right)$

THEOREM 7. For any fixed a large enough, the equation

$$
\left.\operatorname{Re}\left(S_{ \pm 1, \beta}^{\delta}(E)\right)\right|_{E=a+i b}:=\operatorname{Re} \int_{ \pm 1}^{\beta} \sqrt{V(x) \pm i \delta-a-i b} d x=0
$$

has a unique solution $b(a)$, and this solution satisfies

$$
b(a)=i \frac{1}{\beta- \pm 1} \operatorname{Im}(Y(\beta)-Y( \pm 1)) \pm i \delta+O\left(\frac{1}{a}\right) .
$$

Proof. The proof is the same as in Theorem 4 where the potential is $V \pm i \delta$ in each side; we just need to change the interval for the integral. $\mathbf{\square}$

Proof of Theorem 6. The reverse implication is already proved in Theorem 2. Let us prove the direct implication as in the proof of Theorem 3. There exist four WKB solutions $w_{ \pm}^{l, r}$ of $H_{\delta}^{h}-E=0$ on the interval $[-1, \beta]$ or $[\beta, 1]$

$$
\begin{aligned}
& w_{ \pm}^{l}=\left(\partial_{x} z_{l}(x)\right)^{-\frac{1}{2}} e^{ \pm z_{l}(x) / h} W_{ \pm}^{l}\left(z_{l}(x)\right) \\
& w_{ \pm}^{r}=\left(\partial_{x} z_{r}(x)\right)^{-\frac{1}{2}} e^{ \pm z_{r}(x) / h} W_{ \pm}^{r}\left(z_{r}(x)\right)
\end{aligned}
$$

with initial data

$$
\left\{\begin{array}{l}
W_{0, \pm}^{l, r}=1, \\
W_{n,+}^{l}\left(z_{l}(\beta)\right)=0, W_{n,-}^{r}\left(z_{r}(\beta)\right)=0, \quad n \geq 1, \\
W_{n,-}^{l}\left(z_{l}(\beta)\right)=0, W_{n,+}^{r}\left(z_{r}(\beta)\right)=0, \quad n \geq 1 .
\end{array}\right.
$$


Recall that $E \in \operatorname{Sp}\left(H_{\delta}^{h}\right)$ if and only if

$$
\begin{aligned}
& 0=\operatorname{det}\left(\begin{array}{llll}
w_{-}^{l}(-1) & w_{+}^{l}(-1) & 0 & 0 \\
w_{-}^{l}(\beta) & w_{+}^{l}(\beta) & -w_{-}^{r}(\beta) & -w_{+}^{r}(\beta) \\
w^{l^{\prime}}(\beta) & w^{l^{\prime}}(\beta) & -w_{+}^{r \prime}(\beta) & -w_{+}^{r \prime}(\beta) \\
0 & 0 & w_{-}^{r}(1) & w_{+}^{r}(1)
\end{array}\right)= \\
& \frac{1}{h} \partial_{x} z_{r}^{-\frac{1}{2}}(1) \partial_{x} z_{l}^{-\frac{1}{2}}(-1) \times
\end{aligned}
$$

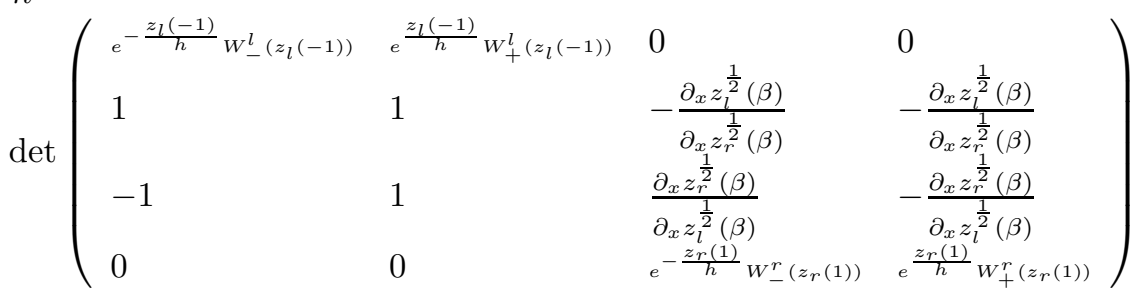

To make the computation, simplify the notation by setting

$$
t=\frac{\partial_{x} z_{l}^{\frac{1}{2}}(\beta)}{\partial_{x} z_{r}^{\frac{1}{2}}(\beta)}, y=e^{\frac{z_{r}(1)}{h}} x=e^{\frac{z_{l}(-1)}{h}}
$$

then (36) becomes

(37) $\operatorname{det}\left(\begin{array}{llll}x^{-1} W_{-}^{l}\left(z_{l}(-1)\right) & x W_{+}^{l}\left(z_{l}(-1)\right) & 0 & 0 \\ 1 & 1 & -t & -t \\ -1 & 1 & t^{-1} & -t^{-1} \\ 0 & 0 & y W_{-}^{r}\left(z_{r}(1)\right) & y^{-1} W_{+}^{r}\left(z_{r}(1)\right)\end{array}\right)=0$,

which gives

$$
x^{2} \frac{W_{+}^{l}\left(z_{l}(-1)\right)}{W_{-}^{l}\left(z_{l}(-1)\right)}=\left(1+y^{2} \frac{W_{-}^{r}\left(z_{r}(1)\right)}{W_{+}^{r}\left(z_{r}(1)\right)} \frac{1-t^{2}}{1+t^{2}}\right)\left(\frac{1-t^{2}}{1+t^{2}}+y^{2} \frac{W_{-}^{r}\left(z_{r}(1)\right)^{-1}}{W_{+}^{r}\left(z_{r}(1)\right)}\right) .
$$

We remark that

$$
\left|\left(1-\frac{\partial_{x} z_{l}(\beta)}{\partial_{x} z_{r}(\beta)}\right)\left(1+{\frac{\partial_{x} z_{l}(\beta)}{\partial_{x} z_{r}(\beta)}}^{-1}\right)\right| \leq C_{8} \sqrt{\delta}
$$

So the condition for $E$ to be in the spectrum of $H_{\delta}^{h}$ is

$$
\begin{aligned}
& \left(e^{2 \frac{z_{l}(-1)}{h}} \frac{W_{+}^{l}\left(z_{l}(-1)\right)}{W_{-}^{l}\left(z_{l}(-1)\right)}-\frac{\left(\partial_{x} z_{r}(\beta)-\partial_{x} z_{l}(\beta)\right)}{\left(\partial_{x} z_{r}(\beta)+\partial_{x} z_{l}(\beta)\right)}\right) \times \\
& \left(e^{-2 \frac{z_{r}(1)}{h}} \frac{W_{-}^{r}\left(z_{r}(1)\right)}{W_{+}^{r}\left(z_{r}(1)\right)}+\frac{\left(\partial_{x} z_{r}(\beta)-\partial_{x} z_{l}(\beta)\right)}{\left(\partial_{x} z_{r}(\beta)+\partial_{x} z_{l}(\beta)\right)}\right) \\
& =\left(1-\frac{\left(\partial_{x} z_{r}(\beta)-\partial_{x} z_{l}(\beta)\right)^{2}}{\left(\partial_{x} z_{r}(\beta)+\partial_{x} z_{l}(\beta)\right)^{2}}\right) .
\end{aligned}
$$


We write this condition by taking logs as

$$
\begin{aligned}
& \ln \left(e^{2 \frac{z_{l}(-1)}{h}} \frac{W_{+}^{l}\left(z_{l}(-1)\right)}{W_{-}^{l}\left(z_{l}(-1)\right)}-\frac{\left(\partial_{x} z_{r}(\beta)-\partial_{x} z_{l}(\beta)\right)}{\left(\partial_{x} z_{r}(\beta)+\partial_{x} z_{l}(\beta)\right)}\right) \\
& +\ln \left(e^{-2 \frac{z_{r}(1)}{h}} \frac{W_{-}^{r}\left(z_{r}(1)\right)}{W_{+}^{r}\left(z_{r}(1)\right)}+\frac{\left(\partial_{x} z_{r}(\beta)-\partial_{x} z_{l}(\beta)\right)}{\left(\partial_{x} z_{r}(\beta)+\partial_{x} z_{l}(\beta)\right)}\right) \\
& -\ln \left(1-\frac{\left(\partial_{x} z_{r}(\beta)-\partial_{x} z_{l}(\beta)\right)^{2}}{\left(\partial_{x} z_{r}(\beta)+\partial_{x} z_{l}(\beta)\right)^{2}}\right)=2 i k \pi, \quad k \in \mathbb{Z} .
\end{aligned}
$$

Step1 First assume that

$$
\operatorname{Re}\left(\int_{-1}^{\beta} \sqrt{V-i \delta-E_{0}} d x\right)=0 \text { and } \operatorname{Re}\left(\int_{\beta}^{1} \sqrt{V+i \delta-E_{0}} d x\right) \neq 0 .
$$

(The case $\operatorname{Re}\left(\int_{-1}^{\beta} \sqrt{V-i \delta-E_{0}} d x\right) \neq 0$ and $\operatorname{Re}\left(\int_{\beta}^{1} \sqrt{V+i \delta-E_{0}} d x\right)=0$ is treated the same way. )

As (30), we get

$$
\left|\ln W_{+}^{l}\left(z_{l}(-1)\right)\right|+\left|\ln W_{-}^{l}\left(z_{l}(-1)\right)\right| \leq C_{1}\left|E_{0}\right|^{-\frac{3}{2}}
$$

for $\left|E-E_{0}\right| \leq C_{3} h \sqrt{\left|E_{0}\right|}$ and $E_{0}>C$ big, $h$ small. As in (26), we get

$$
\left|\ln W_{+}^{r}\left(z_{r}(1)\right)\right|+\left|\ln W_{-}^{r}\left(z_{r}(1)\right)\right| \leq C h
$$

with $C$ uniform in $E_{0}$, for $\left|E-E_{0}\right| \leq C_{3} h \sqrt{\left|E_{0}\right|}$ and

$$
\left|e^{-2 \frac{z_{r}(1)}{h}}\right| \leq e^{-\frac{C}{h}}
$$

Rewrite the previous condition as

$$
\begin{aligned}
& g_{1, k}(E)=2 z_{l}(-1)+h \ln \left(\frac{W_{+}^{l}\left(z_{l}(-1)\right)}{W_{-}^{l}\left(z_{l}(-1)\right)}\right) \\
& +h \ln \left(1-e^{-2 \frac{z_{l}(-1)}{h}} \frac{W_{-}^{l}\left(z_{l}(-1)\right)}{W_{+}^{l}\left(z_{l}(-1)\right)} \frac{\left(\partial_{x} z_{r}(\beta)-\partial_{x} z_{l}(\beta)\right)}{\left(\partial_{x} z_{r}(\beta)+\partial_{x} z_{l}(\beta)\right)}\right) \\
& +h \ln \left(\frac{\left(\partial_{x} z_{r}(\beta)-\partial_{x} z_{l}(\beta)\right)}{\left(\partial_{x} z_{r}(\beta)+\partial_{x} z_{l}(\beta)\right)}\right) \\
& +h \ln \left(1+e^{-2 \frac{z_{r}(1)}{h}} \frac{W_{-}^{r}\left(z_{r}(1)\right)}{W_{+}^{r}\left(z_{r}(1)\right)} \frac{\left(\partial_{x} z_{r}(\beta)+\partial_{x} z_{l}(\beta)\right)}{\left(\partial_{x} z_{r}(\beta)-\partial_{x} z_{l}(\beta)\right)}\right) \\
& -h \ln \left(1-\frac{\left(\partial_{x} z_{r}(\beta)-\partial_{x} z_{l}(\beta)\right)^{2}}{\left(\partial_{x} z_{r}(\beta)+\partial_{x} z_{l}(\beta)\right)^{2}}\right)-2 i k h \pi=0 .
\end{aligned}
$$

Setting

$$
f_{1}(E)=2 z_{l}(-1)+2 \operatorname{Im}\left(\int_{-1}^{\beta} \sqrt{V(x)-i \delta-E_{0}} d x\right)
$$

and

$$
k_{0}=\left\lfloor\operatorname{Im}\left(\int_{-1}^{\beta} \sqrt{V(x)-i \delta-E_{0}} d x\right) h^{-1}\right\rfloor,
$$


we have $f_{1}\left(E_{0}\right)=0$. Using (40), (41) and (42) gives

$$
\left|g_{1, k_{0}}(E)-f_{1}(E)\right| \leq h\left(1+C \sqrt{\delta}+C h \sqrt{\delta}^{-1}+C_{1}\left|E_{0}\right|^{-\frac{3}{2}}\right) .
$$

Now recall that

$$
\left|f_{1}(E)-f_{1}\left(E_{0}\right)\right| \geq \frac{1}{\sqrt{\left|E_{0}\right|}}\left|\left(E-E_{0}\right)\right| .
$$

We can apply the Rouché's Theorem to prove the existence of an eigenvalue of $H_{\delta}^{h}$ for each $h$ at a distance $C_{3}(\delta) \sqrt{\left|E_{0}\right|} h$ of $E_{0}$ for $E_{0}$ large.

Step2 Assume now that

$$
\operatorname{Re}\left(\int_{-1}^{\beta} \sqrt{V-i \delta-E_{0}} d x\right)=\operatorname{Re}\left(\int_{\beta}^{1} \sqrt{V+i \delta-E_{0}} d x\right)=0 .
$$

As in (30),

$$
\begin{array}{r}
\left|\ln W_{+}^{l}\left(z_{l}(-1)\right)\right|+\left|\ln W_{-}^{l}\left(z_{l}(-1)\right)\right|+\left|\ln W_{+}^{r}\left(z_{r}(1)\right)\right|+ \\
\left|\ln W_{-}^{r}\left(z_{r}(1)\right)\right| \leq C_{9}\left|E_{0}\right|^{-\frac{3}{2}} .
\end{array}
$$

For $E_{0}>C, C$ sufficiently large, and $\left|E-E_{0}\right| \leq C_{3} h \sqrt{\left|E_{0}\right|}$ with $h$ small. We write (39) as

$$
\begin{aligned}
& g_{2, k}(E)=2 z_{l}(-1)-2 z_{r}(1)+h \ln \left(\frac{W_{+}^{l}\left(z_{l}(-1)\right)}{W_{-}^{l}\left(z_{l}(-1)\right)}\right) \\
& +h \ln \left(1-e^{-2 \frac{z_{l}(-1)}{h}} \frac{W_{-}^{l}\left(z_{l}(-1)\right)}{W_{+}^{l}\left(z_{l}(-1)\right)} \frac{\left(\partial_{x} z_{r}(\beta)-\partial_{x} z_{l}(\beta)\right)}{\left(\partial_{x} z_{r}(\beta)+\partial_{x} z_{l}(\beta)\right)}\right) \\
& h \ln \left(\frac{W_{+}^{r}\left(z_{r}(1)\right)}{W_{-}^{r}\left(z_{r}(1)\right)}\right)+h \ln \left(1-e^{2 \frac{z_{r}(1)}{h}} \frac{W_{-}^{r}\left(z_{r}(1)\right)}{W_{+}^{r}\left(z_{r}(1)\right)} \frac{\left(\partial_{x} z_{r}(\beta)-\partial_{x} z_{l}(\beta)\right)}{\left(\partial_{x} z_{r}(\beta)+\partial_{x} z_{l}(\beta)\right)}\right) \\
& -h \ln \left(1-\frac{\left(\partial_{x} z_{r}(\beta)-\partial_{x} z_{l}(\beta)\right)^{2}}{\left(\partial_{x} z_{r}(\beta)+\partial_{x} z_{l}(\beta)\right)^{2}}-2 i k h \pi=0,\right.
\end{aligned}
$$

and write

$$
\begin{aligned}
f_{2}(E)=2 z_{l}(-1)-2 z_{r}(1)- & 2 \operatorname{Im}\left(\int_{\beta}^{-1} \sqrt{V(x)-i \delta-E_{0}} d x\right) \\
& +2 \operatorname{Im}\left(\int_{\beta}^{1} \sqrt{V(x)+i \delta-E_{0}} d x\right) .
\end{aligned}
$$

Let

$$
k_{0}=\left\lfloor\operatorname{Im}\left(\int_{\beta}^{-1} \sqrt{V(x)-i \delta-E_{0}} d x-\int_{\beta}^{1} \sqrt{V(x)+i \delta-E_{0}} d x\right) h^{-1}\right\rfloor .
$$

Then

$$
\left|f_{2}(E)-f_{2}\left(E_{0}\right)\right| \geq{\sqrt{\left|E_{0}\right|}}^{-1}\left|\left(E-E_{0}\right)\right|
$$


and for $\left|E-E_{0}\right| \leq C_{3} h \sqrt{\left|E_{0}\right|}$

$$
\left|f_{2}(E)-g_{2, k_{0}}(E)\right| \leq h\left(C_{9}\left|E_{0}\right|^{-\frac{3}{2}}+C_{8} \sqrt{\delta}+1\right) .
$$

On the set where $\left|E-E_{0}\right|=C_{3} h \sqrt{\left|E_{0}\right|}$, we have for $C_{3}=C_{3}(\delta)$ and $E_{0}$ large

$$
\left|\left(f_{2}-g_{2, k_{0}}\right)(E)\right|<\left|f_{2}(E)\right| .
$$

All the hypotheses of Rouché's theorem are now satisfied and we conclude as before.

6. Application to the harmonic oscillator. In this section we are interested in the Dirichlet eigenvalues of the operator $P^{h}$ on $L^{2}([-1,1])$,

$$
P^{h}=-h^{2} \frac{d}{d x^{2}}+i x^{2}, \quad\left\{\begin{array}{l}
P^{h} u=E u \\
u(-1)=u(1)=0 .
\end{array}\right.
$$

We obtain here only two results. Theorem 10 describes the shape of the set $\lim _{h} \operatorname{Sp}\left(P^{h}\right)$, cf. figure 14. Theorem 12 shows how the spectrum changes near $\infty$ when $P^{h}$ is change by a specific perturbation of size $\delta$, as illustrated in figure 15 .

For any $E \in \mathbb{C}^{*}$ there are two turning points, $\alpha_{ \pm}(E)= \pm(-i E)^{1 / 2}$, which are both simple i.e. of order 1 .

Denote by $S_{x, y}(E)=\int_{x}^{y}\left(i t^{2}-E\right)^{1 / 2} d t$. The function $S_{0, x}$ is even, so to simplify the computation one can use that $S_{-x, x}=2 S_{0, x}$. Writing

$$
\begin{gathered}
z(x, y, E)=\int_{x}^{y}\left(i t^{2}-E\right)^{1 / 2} d t, \\
z^{\text {har }}(x, y, E)=\int_{x}^{y}\left(u^{2}-E\right)^{1 / 2} d u,
\end{gathered}
$$

and changing coordinate $t=e^{-\frac{i \pi}{8}} u$, we get

$$
z(x, y, E)= \pm z^{h a r}\left(x e^{\frac{i \pi}{8}}, y e^{\frac{i \pi}{8}}, E e^{-\frac{i \pi}{4}}\right) .
$$

So the Stokes lines of $-h^{2} \Delta+i x^{2}$ can be deduced from those of the harmonic oscillator $-h^{2} \Delta+x^{2}$ by a rotation by $-\frac{\pi}{8}$ about the origin, [12]. Using the geometry of the Stokes lines we find for some $E$ a progressive path from -1 to 1 . Combine this with previous theorems, we obtain a partial description of $\mathcal{T}^{c}$ :

THEOREM 8. We have

- Suppose $E \in \mathbb{C}^{*}$ is such that $\operatorname{Re} S_{\alpha_{-}, \alpha_{+}}(E) \neq 0, \operatorname{Re} S_{-1,1}(E) \neq 0$, $\operatorname{Re} S_{\alpha_{+}, 1}(E) \neq 0, \operatorname{Re} S_{\alpha_{-}, 1}(E) \neq 0$, then $E \in \mathcal{T}_{0}$

- If $E \in \mathcal{T}_{0}$, then for any $h$ small enough, $E$ is not an eigenvalue for $P^{h}$.

- If $E \in \lim _{h} \operatorname{Sp}\left(P^{h}\right)$ then $E \in \mathcal{T}_{0}^{c}$

Proof. Using Theorem 1 and Theorem 2, it is enough to find a path $\gamma$ transversal to the Stokes lines from -1 to 1 . We have $\operatorname{Re} S_{\alpha_{-}, \alpha_{+}}(E) \neq 0$, so the Stokes line issuing from $\alpha_{+}$do not intersect the Stokes lines issuing form $\alpha_{-}$. Therefore the complex plane is divided into exactly five region delimited by the Stokes lines issuing from the turning points. In Figure 1, we have drawn the configuration of the Stokes lines up to an analytic diffeomorphism. 


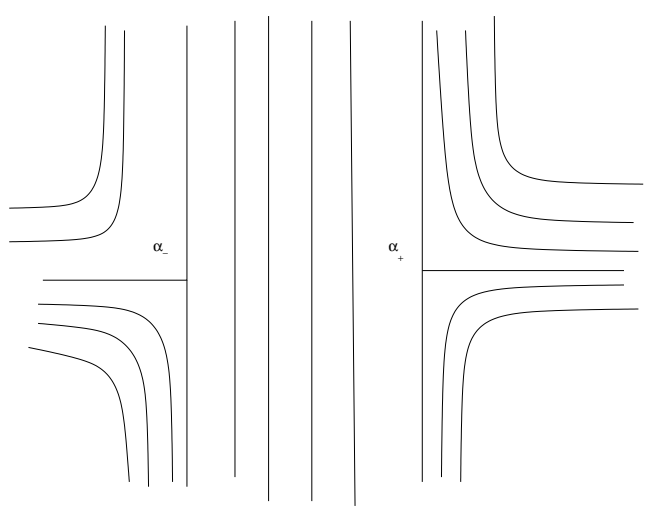

FIG. 1. Non degenerate Stokes lines

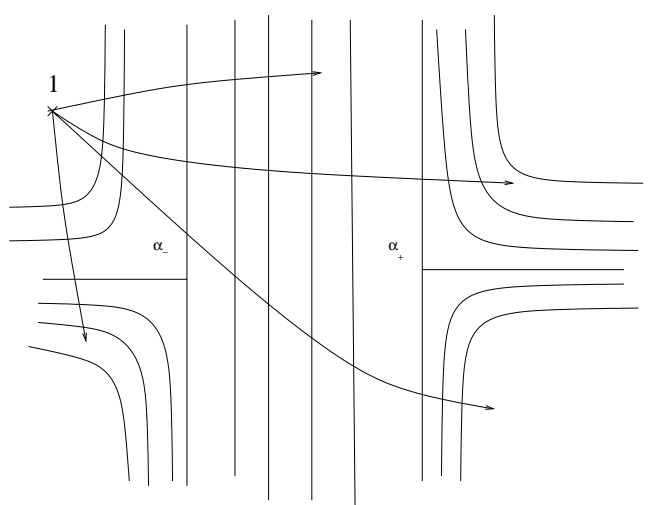

FIG. 2. Progressive path issuing from 1

We have $\operatorname{Re} S_{1, \alpha_{ \pm}}(E) \neq 0$, so that in particular 1, (and by symmetry -1 ) are not on any boundary of the Stokes regions.

If 1 and -1 belong to different regions, then there always exists a path $\gamma$ transversal to the Stokes lines (see the figure 2); if on the other hand 1 and -1 belong to the same region, but we also have $\operatorname{Re} S_{-1,1}(E) \neq 0$, then they do not belong to the same Stokes lines and there still exists such a path $\gamma$.

If $\operatorname{Re} S_{\alpha_{-}, \alpha_{+}}(E)=0$, the Stokes configuration is as in Figure 3 .

We now picture the evolution of the Stokes lines as $E$ moves in the complex plane.

In fact Theorem 8 can be improved. Choose the segment $\left[\alpha_{-}, \alpha_{+}\right]$as a cut for $x \mapsto(V(x)-E)^{1 / 2}$. The pictures 5 and 6 show the branch of the square root for different values of $E$.

Denote by

$$
\Gamma_{-1,1}=\left\{E \in \mathbb{C} ; \operatorname{Re} S_{-1,1}(E)=0, \quad \operatorname{Re} S_{\alpha_{-}, \alpha_{+}}(E) \neq 0\right\}
$$

The next theorem is proved by listing all the situations where there do not exist progressive paths from -1 to 1 using the geometry of the Stokes lines. 


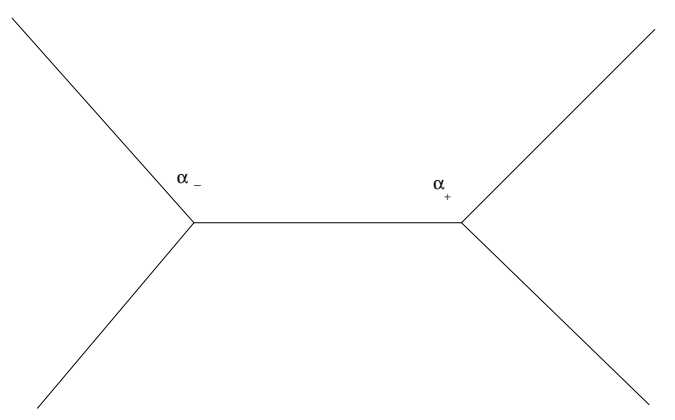

FIG. 3. Degenerate Stokes lines

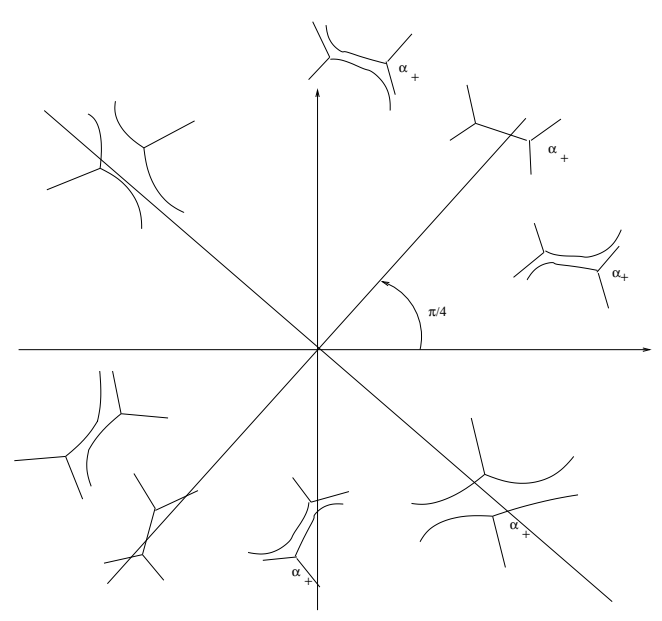

FIG. 4. Stokes line for different $E$

TheOREM 9. We have

- Suppose $E \in \mathbb{C}^{*}$ belongs to

$$
\Gamma_{\alpha_{-}, \alpha_{+}}=\left\{E \in \mathbb{C} ; \operatorname{Re} S_{\alpha_{-}, \alpha_{+}}(E)=0, \operatorname{Re} S_{\alpha_{+}, 1}(E) \leq 0\right\},
$$

then $E \in \mathcal{T}_{0}$.

- Suppose $E \in \mathbb{C}^{*}$ belong to

$$
\Gamma_{\alpha_{+}, 1}=\left\{E \in \mathbb{C} ; \operatorname{Re} S_{\alpha_{+}, 1}(E)=0 ; \operatorname{Im} S_{\alpha_{+}, 1}(E) \leq 0\right\},
$$

then $E \in \mathcal{T}_{0}$.

- Suppose $E \in \mathbb{C}^{*}$ belong to

$$
\Gamma_{\alpha_{-}, 1}=\left\{E \in \mathbb{C} ; \operatorname{Re} S_{\alpha_{-}, 1}(E)=0 ; \operatorname{Im} S_{\alpha_{-}, 1}(E) \geq 0\right\},
$$

then $E \in \mathcal{T}_{0}$.

- $E \notin \Gamma_{\alpha_{+}, 1} \cup \Gamma_{\alpha_{-}, 1} \cup \Gamma_{\alpha_{-}, \alpha_{+}} \cup \Gamma_{-1,1}$ then there is no progressive path form -1 to 1 .

REMARK 6. If a progressive path enters a Stokes region crossing a Stokes line issuing from a turning point $\alpha$, then it cannot leave this region by crossing any others Stokes lines issuing from the same turning point $\alpha$. 


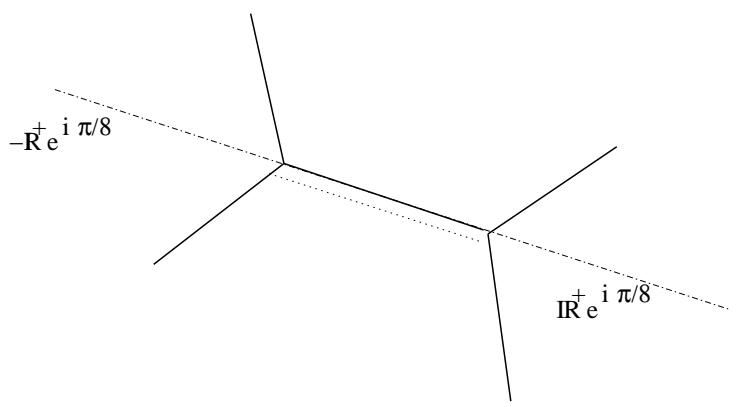

FIG. 5. branch for $E=e^{i \frac{\pi}{4}}$, the cut is the dashed line and also a Stokes line

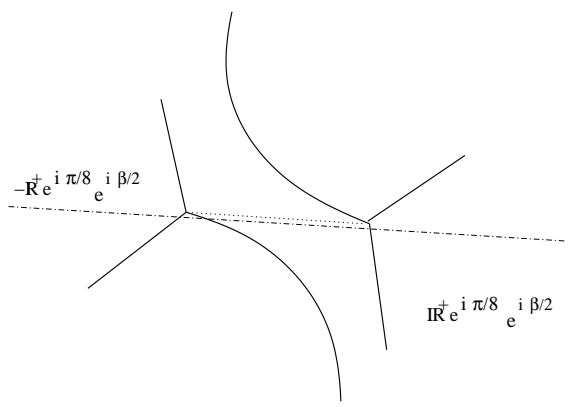

FIG. 6. branch for $E=e^{i \frac{\pi}{4}} e^{i \beta}, \beta>0$ small. The cut is the dashed line

The proof consists in listing the cases where progressive paths do not exist. Recall first that if 1 and -1 belong to the same region then there exists a progressive path if and only if $\operatorname{Re} S_{-1,1}(E) \neq 0$. We get $\Gamma_{-1,1}=\{E \in \mathbb{C} ; 1$ and 1 belong to the same region $\left.\operatorname{Re} S_{-1,1}(E)=0\right\}$. Now assuming 1 and -1 belong to different regions, we get two different figures for the Stokes lines either $\operatorname{Re} S_{\alpha_{-}, \alpha_{+}}(E)=0$ (Figure3) or $\operatorname{Re} S_{\alpha_{-}, \alpha_{+}}(E) \neq 0$ (Figure 1). In the first case, using Remark 6, we see that we cannot find a path if and only if 1 belongs to the hatched region ( and -1 by symmetry to the opposite one)(Figure 7 ).

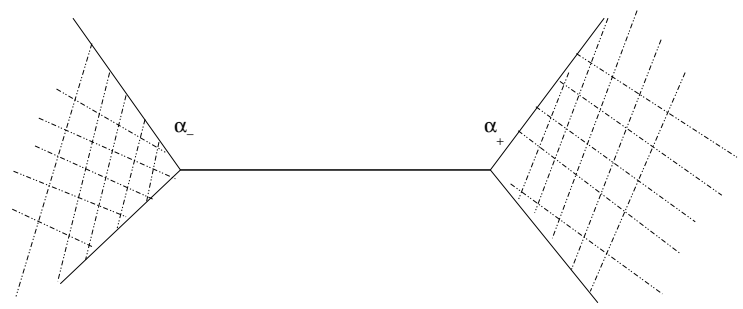

FIG. 7. The position of 1 is in one of hatched regions

The condition $\operatorname{Re} S_{\alpha_{-}, \alpha_{+}}(E)=0, \quad \operatorname{Re} S_{\alpha_{+}, 1}(E)>0$ corresponds to this situation, i.e. Figure 8 shows the sign of the quantity $\operatorname{Re} S_{\alpha_{+}, x}\left(e^{i \frac{\pi}{4}} \lambda\right)$ depends on the position of $x$ for $\lambda \in \mathbb{R}$.

Now assuming 1 and -1 belong to different regions and $\operatorname{Re} S_{\alpha_{-}, \alpha_{+}}(E) \neq 0$ (Figure 6 ). Using Remark 6, we see that we cannot find a path if and only if 1 belong the dotted curves ( and -1 by symmetry to the opposite one )(Figure 9 ). The condition 


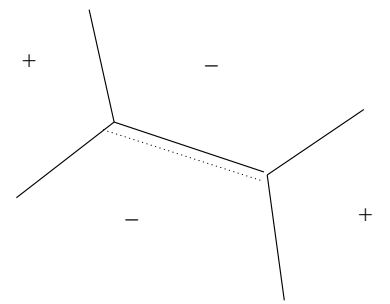

FIG. 8. Sign of the quantity $x \rightarrow \operatorname{Re} S_{\alpha_{+}, x}\left(e^{i \frac{\pi}{4}}\right)$

$\operatorname{Re} S_{\alpha_{+}, 1}(E)=0, \quad \operatorname{Im} S_{\alpha_{+}, 1}(E)>0$ correspond to Figure 9.

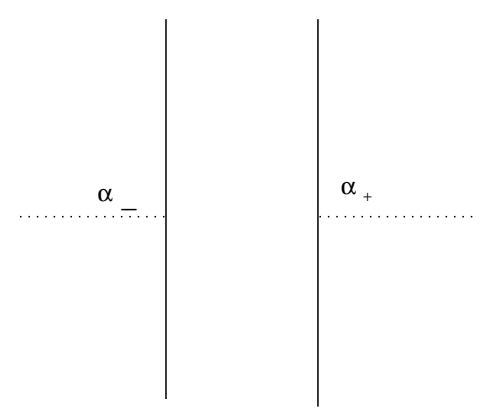

FIG. 9. The position of 1 is in one of the dotted line, that are Stokes lines

The condition $\operatorname{Re} S_{\alpha_{+}, 1}(E)=0, \quad \operatorname{Im} S_{\alpha_{+}, 1}(E)>0$ corresponds to this case. i.e. Figure 10 shows the sign of the quantity $\operatorname{Im} S_{\alpha_{ \pm}, x}\left(e^{i \frac{\pi}{4}} e^{i \beta} \lambda\right)$ with $\beta>0$ and small depending on $x$ near to $\alpha_{ \pm}$.

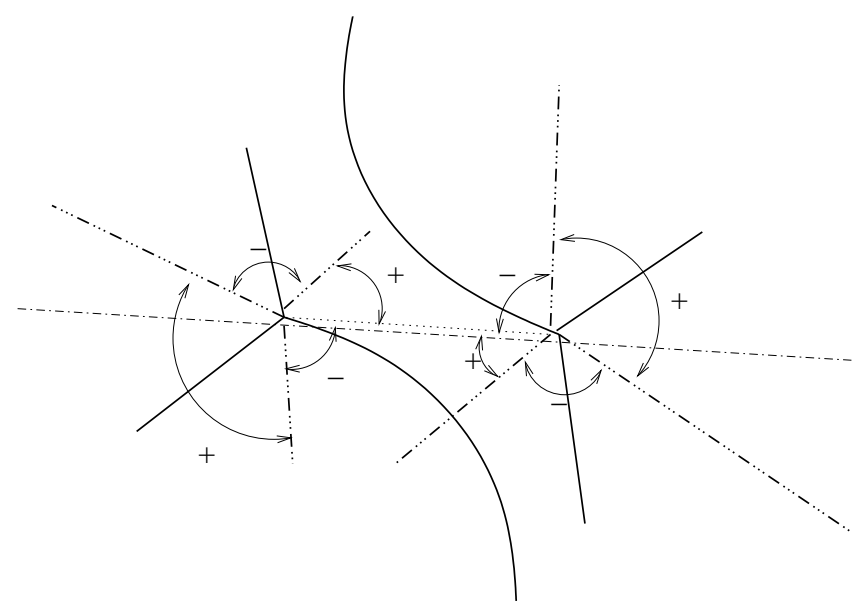

FIG. 10. Sign of the quantity $\operatorname{Im} S_{\alpha_{ \pm}, x}\left(e^{i \frac{\pi}{4}} \lambda e^{i \beta}\right)$

Now we want to describe the set $\mathcal{T}_{0}^{c}$. 
THEOREM 10. We have

1. The set $\mathcal{T}_{0}^{c}$ is the union of three curves $\Gamma_{\alpha_{+}, 1}, \Gamma_{\alpha_{-}, \alpha_{+}}, \Gamma_{-1,1}$ with only one of infinite length $\Gamma_{-1,1}$.

2. The three curves meet at a common point $\lambda_{0} e^{\frac{i \pi}{4}}$ with $\lambda_{0} \in \mathbb{R}^{+}$.

3. The curve $\Gamma_{-1,1}$ goes from $\lambda_{0} e^{\frac{i \pi}{4}}$ to $\mathbb{R}+\frac{i}{3}$.

4. $\Gamma_{\alpha_{-}, \alpha_{+}}=\left\{\lambda e^{\frac{i \pi}{4}} ; \lambda \in \mathbb{R}, 0 \leq \lambda<\lambda_{0}\right\}$.

5. The curve $\Gamma_{\alpha_{+}, 1}$ goes from $i$ to $\lambda_{0} e^{\frac{i \pi}{4}}$.

Proof. (1) :

Let $E=\lambda^{2} e^{i \beta}$ with $\beta \in \mathbb{R}$ and $\lambda \in \mathbb{R}$. We fix $\beta$ and increase $\lambda$. We get $z\left(x, y, \lambda^{2} e^{i \beta}\right)=z\left(\frac{x}{\lambda}, \frac{y}{\lambda}, e^{i \beta}\right)$. The shape of the Stokes lines remains invariant up to dilation by $\lambda$. Let $\beta$ be such that $\operatorname{Re} S_{\alpha_{-} . \alpha_{+}}\left(e^{i \beta}\right) \neq 0$ then under symmetry we get $\operatorname{Re} S_{0, \alpha_{+}}\left(e^{i \beta}\right) \neq 0$, and $\operatorname{Re} S_{\alpha_{-} .0}\left(e^{i \beta}\right) \neq 0$. So if $\beta$ is such that there is no Stokes line issuing from the turning points and going through 0 , then there exists a neighborhood of 0 with no point of any Stokes lines issuing from the turning points. For $\lambda$ big enough, we get that -1 and 1 belong to this neighborhood of 0 . Then $E$ will belong to $\mathcal{T}_{0}^{c}$ if and only if $\operatorname{Re} S_{-1,1}(E) \neq 0$.

If $\beta$ is such that $\operatorname{Re} S_{\alpha_{-}, \alpha_{+}}\left(e^{i \beta}\right)=0$ then $\beta= \pm \frac{\pi}{4}$ and Figure 12 and the fact that the spectrum is included in the set $\left\{E \in \mathbb{C} ; E=\mathbb{R}^{+}+i x^{2}, x \in[-1,1]\right\}$ (i.e. the pseudo spectrum or the values of the principal symbol) shows that $E \in \mathcal{T}_{0}^{c}, E=\lambda e^{i \frac{\pi}{4}}$, $\lambda \in \mathbb{R}$, implies $E$ bounded. So we have that if $E$ is big enough and belongs to $\mathcal{T}_{0}^{c}$, then $E \in \Gamma_{-1,1}$.

We choose the determination of $\alpha_{ \pm}$so that the set

$$
\Gamma_{\alpha_{-}, 1}=\left\{E \in \mathbb{C} ; \operatorname{Re} S_{\alpha_{-}, 1}(E)=0 ; \operatorname{Im} S_{\alpha_{-}, 1}(E) \geq 0\right\}
$$

is empty see Figure 4.

The second statement is proved by the relation

$$
S_{0, \alpha_{+}}(E)+S_{\alpha_{+}, 1}(E)+S_{1,0}(E)=0,
$$

and illustrated in Figure 11.
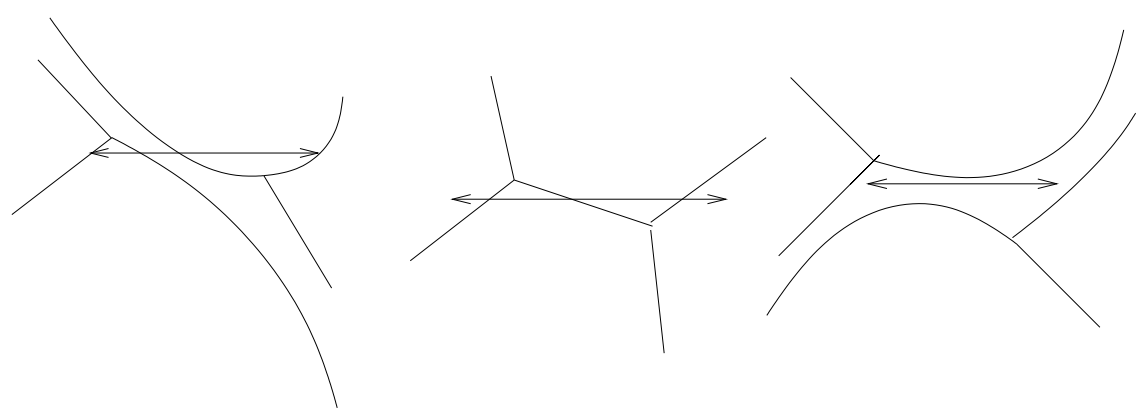

FIG. 11. Stokes line for $E$ near $\lambda_{0} e^{\frac{i \pi}{4}}$, the arrows locate the positions of -1 and 1

Point 3 is proved in Theorem 11 below.

Point 4 is deduced from

$$
z(x, y, E)= \pm \int_{x e^{\frac{i \pi}{8}}}^{y e^{\frac{i \pi}{8}}}\left(u^{2}-E e^{-\frac{i \pi}{4}}\right)^{1 / 2} d u
$$


So for $E=\left\{\lambda e^{\frac{i \pi}{4}}, \lambda \in \mathbb{R}\right\}$ we obtain

$$
\operatorname{Re} S_{0, \alpha_{+}}(E)=\operatorname{Re} \pm \int_{0}^{ \pm \sqrt{\lambda}}\left(u^{2}-\lambda\right)^{1 / 2} d u=0 .
$$

The existence of $\lambda_{0}$ is obvious in Figure 13.

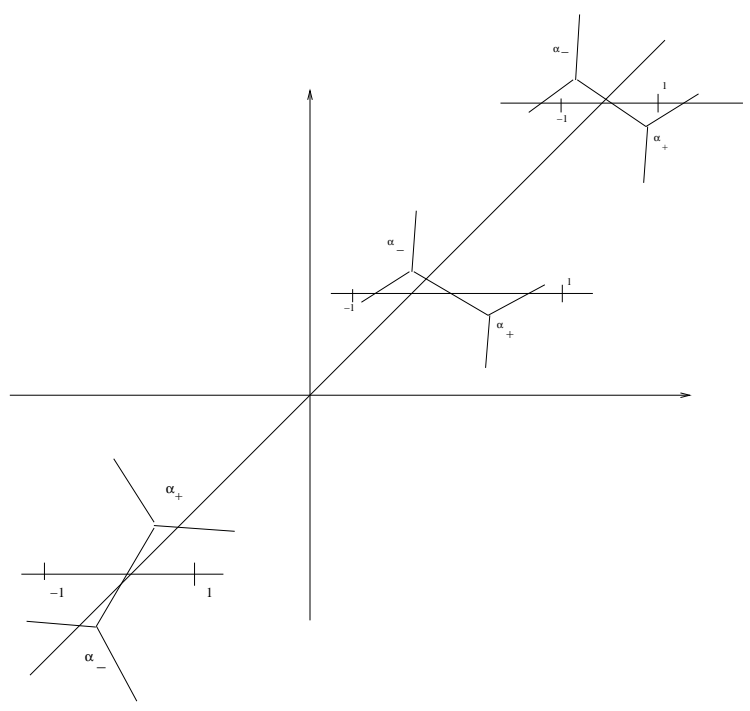

FIG. 12. Stokes line for $E=\lambda e^{\frac{i \pi}{4}}$

Point 5 is obvious from Figure 13.
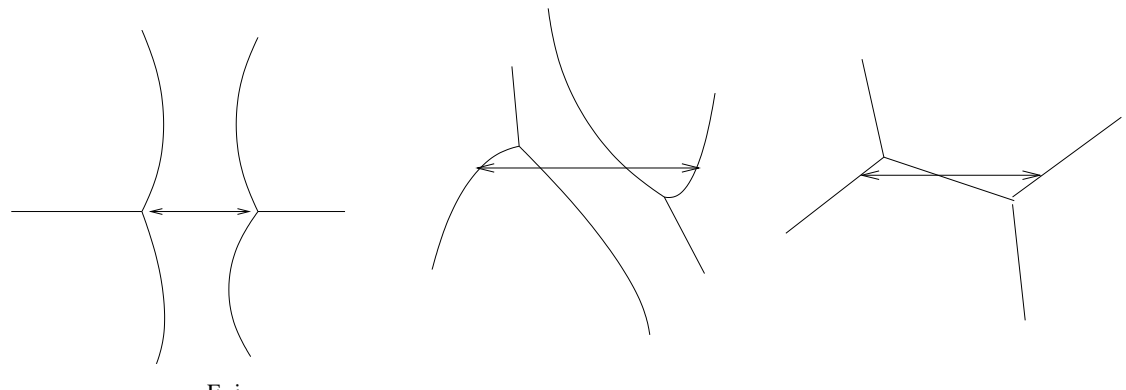

FIG. 13. Evolution of the Stokes line for $E \in \Gamma_{\alpha_{+}, 1}$, the arrows locate the position of -1 and 1

The hypothesis (H1) is obviously satisfied by the potential $V(x)=i x^{2}$. The hypothesis (H2) (H3) are satisfied by the potential $i x^{2}$ ( and by any potential $e^{d} x^{2}$ if $\left.e^{\frac{d}{2}} \notin \mathbb{R}\right)$ but not by the potential $x^{2}$.

For large energies, we summarize the result of Proposition 2, Theorem 3 and Theorem 4 in

Theorem 11. We have

1. Large values of $\lim _{h} \mathrm{Sp} P^{h}$ are close to the curve $\Gamma_{-1,1}$. 
2. The curve $\Gamma_{-1,1}=\left\{E \in \mathbb{C}, \operatorname{Re} S_{-1,1}(E)=0\right\}$ tends to infinity, and is asymptotic to the line $\mathbb{R}+i / 3$.

One could compute the set $\mathcal{T}_{0}^{c}$ with Matlab or Mathematica. In Figure 14 we have drawn the set $\mathcal{T}_{0}^{c}$ using Theorems 10 and 11.

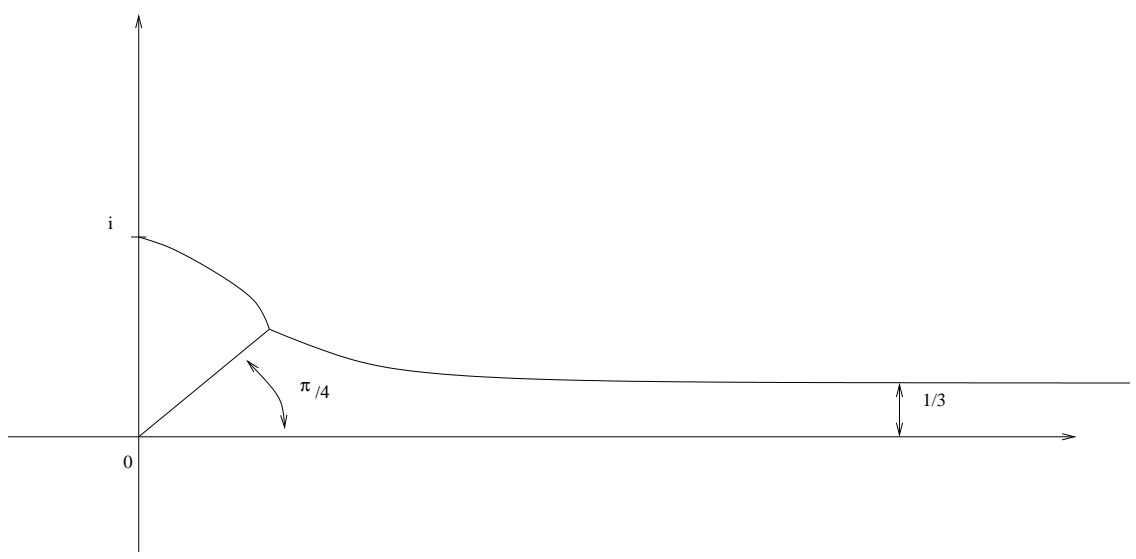

FIG. 14. Limit spectrum of $P^{h}$

For large energies and perturbation, we summarize the results of Theorem 6 and Theorem 7 in

THEOREM 12. We have

1. Large $E \in \lim _{h} \operatorname{Sp} P_{\delta}^{h}$ are near to the union of the two curves $\Gamma_{-1, \beta}, \Gamma_{\beta, 1}$.

2. The curve $\Gamma_{-1, \beta}=\left\{E \in \mathbb{C}, \operatorname{Re} S_{-1, \beta}(E)=0\right\}$ tends to infinity, and is asymptotic to $\mathbb{R}+i \frac{1}{3}\left(\beta^{2}-\beta+1\right)-i \delta$.

3. The curve $\Gamma_{\beta, 1}=\left\{E \in \mathbb{C}, \operatorname{Re} S_{\beta, 1}(E)=0\right\}$ goes to infinity, and is asymptotic to $\mathbb{R}+i \frac{1}{3}\left(\beta^{2}+\beta+1\right)+i \delta$.

We remark that the two curves $\Gamma_{-1, \beta} \Gamma_{\beta, 1}$ are distinct for $\beta \neq 0$.

Figure 15 represents the two Theorems, 11 and 12.

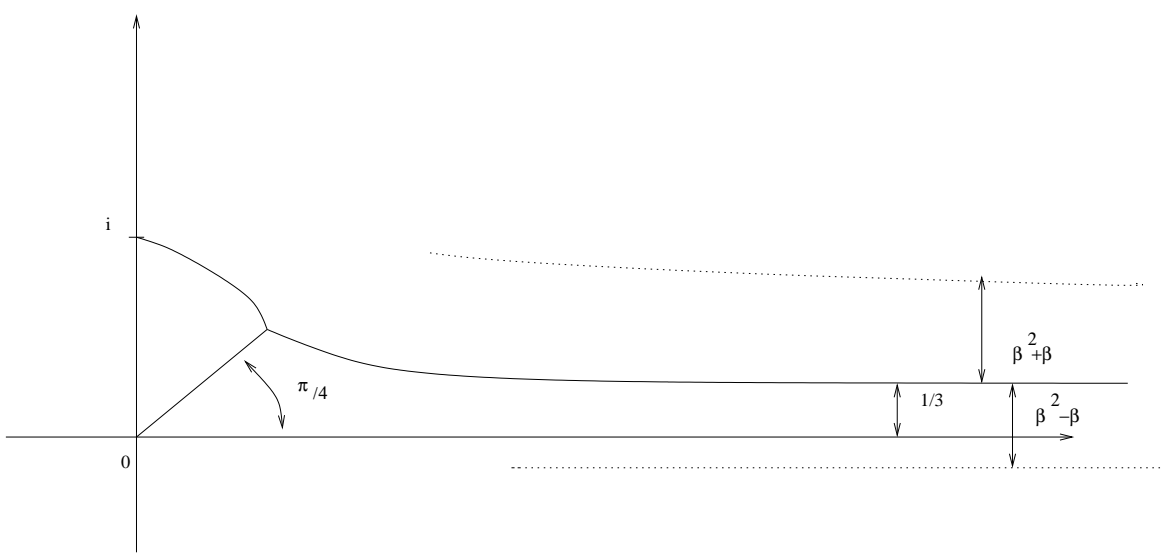

FIG. 15. Spectrum of $P^{h}$ in plain lines and spectrum of $P_{\delta}^{h}$ for $\delta$ small near to $\infty$ as dotted lines. 
7. Pseudo-spectrum. We have defined here the pseudo-spectrum of $H_{0}^{h}$ as the set

$$
\overline{\left\{\xi^{2}+V(x), x \in(-1,1), \xi \in \mathbb{R}, \operatorname{Im}\left(\xi V^{\prime}(x)\right) \neq 0\right\}} .
$$

We remark that for any $z$ is this set, we could construct a function $u^{h}$ in the domain of $H_{0}^{h}$ such that

$$
\left\|\left(H_{0}^{h}-z\right) u^{h}\right\|=\mathcal{O}\left(h^{\infty}\right)\left\|u^{h}\right\| .
$$

Let suppose now that we put two jumps in the potential, one at $\beta$ and one at $\beta^{\prime}$. i.e $V_{\beta \beta^{\prime}}=V+i \delta_{1} H_{\beta}+i \delta_{2} H_{\beta^{\prime}}$.

Assume that $V$ verifies the hypothesis (H1) and :

(H4) If $E$ is large enough, with small enough imaginary part then $1,-1, \beta$ and $\beta^{\prime}$ belong to the same Stokes region.

Then we obtain just as in Theorems 6 and 7 the next two theorems

THEOREM 13. For $E_{0}$ large, the following two conditions are equivalent

$$
\begin{aligned}
& \text { 1. } \operatorname{Re}\left(\int_{-1}^{\beta} \sqrt{V_{\beta \beta^{\prime}}-E_{0}} d x=0\right) \text { or } \operatorname{Re}\left(\int_{\beta^{\prime}}^{1} \sqrt{V_{\beta \beta^{\prime}}-E_{0}} d x=0\right) \text { or } \\
& \operatorname{Re}\left(\int_{\beta}^{\beta^{\prime}} \sqrt{V_{\beta \beta^{\prime}}-E_{0}} d x=0\right) \text {. } \\
& \text { 2. } E_{0} \in \lim _{h} \mathrm{Sp} H_{\delta}^{h} .
\end{aligned}
$$

THEOREM 14. For a large enough, the equation

$$
\left.\operatorname{Re} S_{\beta, \beta^{\prime}}(E)\right|_{E=a+i b}=\operatorname{Re} \int_{\beta}^{\beta^{\prime}} \sqrt{V(x)+i \delta_{1}-a-i b} d x=0
$$

has a unique solution $b(a)$, the solution satisfies

$$
b(a)=i \frac{1}{\beta^{\prime}-\beta} \operatorname{Im}\left(Y\left(\beta^{\prime}\right)-Y(\beta)\right)-i \delta_{1}+O\left(\frac{1}{a}\right) .
$$

We remark that

$$
\lim _{\beta^{\prime} \rightarrow \beta} \frac{1}{\beta^{\prime}-\beta} \operatorname{Im}\left(Y\left(\beta^{\prime}\right)-Y(\beta)\right)=\operatorname{Im}(V(\beta))
$$

This means, letting $\beta^{\prime} \rightarrow \beta$, and $\delta \rightarrow 0$ any values of the form $\left\{\xi^{2}+i \operatorname{Im}(V(x)) ; \xi \in\right.$ $\mathbb{R},|\xi|>>1, x \in[-1,1]\}$ is in the spectrum of this kind of perturbation of $H^{h}$. Thus we can reach all the large values of the pseudo-spectrum with this special type of perturbation.

Acknowledgement. I wish to thank J. Sjöstrand for organizing a working group in IHP, where one of the subjects was pseudo-spectrum. I would also like to thank T. Ramond, who motivated my interest in this problem and helped me to obtain this final presentation, T. Kappeler for useful discussions about the history of Sturm Liouville problems, M. Zworski for pointing out the paper [13]. Finally, I wish to thank the ETH in Zürich where part of this work was done. 


\section{REFERENCES}

[1] Carlson, Robert; Threadgill, Rod; Shubin, Carol Sturm-Liouville eigenvalue problems with finitely many singularities, J. Math. Anal. Appl., 204:1 (1996), pp. 74-101.

[2] Davies, E.B., Pseudo-spectra, the harmonic oscillator, and complex resonances, R. Soc Lond. Proc. Ser. A Math. Phys. Eng. Sci., 455 (1999), pp. 585-599.

[3] Davies, E.B., Non-self-adjoint differential operators, Bull. Lond. Math. Soc., 34:5 (2002), pp. $513-532$.

[4] Davies, E. Brian, Pseudospectra of differential operators, J. Oper. Theory, 43:2 (2000), pp. 243-262.

[5] Dencker, Nils; Sjöstrand, Johannes; Zworski, Maciej, Pseudospectra of semiclassical (pseudo-) differential operators, Comm. Pure Appl. Math., 57:3 (2004), pp. 384-415.

[6] Dyachenko, A.V.; Shkalikov, A.A., On a model problem for the Orr-Sommerfeld equation with linear profile, Funct. Anal. Appl., $36: 3$ (2002), pp. 228-232; translation from, Funkts. Anal. Prilozh., 36:3 (2002), pp. 71-75.

[7] Fujile, S., RAmond, T., Matrice de scattering et résonances associées à une orbite hétérocline, (Scattering matrices and resonances associated to a heteroclinic orbit), Ann. Inst. Henri Poincaré, Phys. Théor, 69:1 (1998), pp. 31-82.

[8] Gérard, C.; Grigis, A., Precise estimates for tunneling and eigenvalues near a potential barrier, J. Differ. Equations, 72:1 (1988), pp. 149-177.

[9] Hitrik, M., Boundary spectral behavior for semiclassical operators in one dimension, Preprint.

[10] Kappeler, T.; MöHR, C., Estimates for periodic and Dirichlet eigenvalues of the Schrödinger operator with singular potentials, J. Funct. Anal., 186:1 (2001), pp. 62-91.

[11] Marchenko, Vladimir A., Sturm-Liouville operators and applications. Transl. from the Russian by A. Iacob, Operator Theory: Advances and Applications, Vol. 22. Basel/Boston/Stuttgart: Birkhuser Verlag. XI, 367 p. DM 148.00 (1986).

[12] Maslov, V. P.; Fedoriuk, M. V., Semiclassical approximation in quantum mechanics, Translated from the Russian by J. Niederle and J. Tolar. Mathematical Physics and Applied Mathematics, 7. Contemporary Mathematics, 5. D. Reidel Publishing Co., DordrechtBoston, Mass., 1981. ix+301 pp. ISBN: 90-277-1219-0.

[13] Miller, Peter D., Some remarks on a WKB method for the nonselfadjoint Zakharov-Shabat eigenvalue problem with analytic potentials and fast phase, Physica D, pp. 152-153, 145$162(2001)$.

[14] Redparth, Paul, Spectral properties of non-self-adjoint operators in the semi-classical regime, J. Diff. Equations, 177:2 (2001), pp. 307-330.

[15] Shkalikov, A.A., Spectral portraits of the Orr-Sommerfeld operator with large reynolds numbers, J. M. sciences, 00 (2000), pp. 110-134.

[16] Shkalikov, A.A., Spectral portraits and the resolvent growth of a model problem associated with the Orr-Sommerfeld equation, arXiv number 0306342. 